\title{
Information-Theoretic Analysis of OFDM with Subcarrier Number Modulation
}

\author{
Shuping Dang, Member, IEEE, Shuaishuai Guo, Member, IEEE, Basem Shihada, Senior Member, IEEE, and \\ Mohamed-Slim Alouini, Fellow, IEEE
}

\begin{abstract}
With the prevalence of orthogonal frequencydivision multiplexing (OFDM) in many standards, e.g., IEEE 802.11, IEEE 802.16, DVB-T, and DVB-T2, a number of variant modulation schemes based on OFDM have been proposed, which resort to signal sparsity to further enhance spectral efficiency and mitigate the high peak-to-average ratio (PAPR) problem. Among these variants, OFDM with subcarrier number modulation (OFDM-SNM) has been proven to be efficient for simple communication systems with low constellation modulation orders and limited decoding capability. To rigorously verify the performance advantages of OFDM-SNM, we present the study of OFDM-SNM in this paper from the information-theoretic perspective. In particular, we determine an upper bound on the mutual information of OFDM-SNM in closed form by using the log sum inequality. Also, we analyze the optimal pattern utilization probabilities (PUPs) for OFDM-SNM by channel-dependent coding and propose an easy-to-implement iterative algorithm to approach the optimal PUPs. Moreover, considering the practical achievability, we propose a Huffman coding based achievable PUP vector construction scheme to obtain the achievable PUPs and the corresponding achievable rate. We carry out numerical simulations to verify the effectiveness of this study and illustrate the efficiency of the obtained PUPs in comparison with several benchmarks.
\end{abstract}

Index Terms-Orthogonal frequency-division multiplexing (OFDM), subcarrier number modulation (SNM), channel capacity, information-theoretic analysis, channel-dependent coding.

\section{INTRODUCTION}

$\mathbf{O}$ RTHOGONAL frequency-division multiplexing (OFDM) has become increasingly prevalent since being adopted as one of the core technologies for fourth generation $(4 \mathrm{G})$ communications [1]. It has been accepted in many widely used wireless and wired network standards, e.g., IEEE 802.11 (WiFi), IEEE 802.16 (WiMAX), DVB$\mathrm{T}$, and DVB-T2 [2], [3]. From the current communication technological developments and trends, OFDM and its variants still dominant the fifth generation (5G) communications and are highly expected to play a key role for beyond 5G (B5G) communications and even sixth generation (6G) communications [4], [5]. To further enhance the performance of classic OFDM and complement its drawbacks, e.g., high peak-to-average power ratio (PAPR), OFDM with index modulation (OFDM-IM), a variant OFDM scheme, is

S. Dang, B. Shihada and M.-S. Alouini are with Computer, Electrical and Mathematical Science and Engineering Division, King Abdullah University of Science and Technology (KAUST), Thuwal 23955-6900, Kingdom of Saudi Arabia (e-mail: \{shuping.dang, basem.shihada, slim.alouini\}@kaust.edu.sa).

S. Guo is with Shandong Provincial Key Laboratory of Wireless Communication Technologies and School of Control Science and Engineering, Shandong University, Jinan 250061, China (email: shuaishuai_guo@sdu.edu.cn). proposed and has been rigorously proven to be more efficient than the classic OFDM scheme under certain conditions [6]. The original proposal of OFDM-IM has sparked the research enthusiasm of frequency-domain sparsity modulation in recent years [7]-[10]. Abundant research works are devoted to find enhanced OFDM-IM schemes with certain peculiarities, novel modulation dimensions, and/or for special application scenarios [11]-[17].

Different from OFDM-IM utilizing the indexes of subcarrier activation pattern (SAP) with a fixed number of active subcarriers to piggyback extra information, another sibling frequency-domain sparsity modulation scheme, termed OFDM with subcarrier number modulation (OFDM-SNM), resorts to the number of active subcarriers to encode extra information [18]. It has been proven in [19] that the transmission rate of OFDM-SNM could be higher than those of plain OFDM and OFDM-IM for simple communication systems with low constellation modulation orders and limited decoding capability. This feature makes OFDM-SNM a promising modulation scheme for the applications in the Internet of Things (IoT) and machine-type communication (MTC) networks [20], [21].

Recently, continuous endeavors are exerted to fit OFDMSNM for different application scenarios with various service requirements. In [22] OFDM-SNM and OFDM-IM are merged to provide a higher degree of design freedom and spectral efficiency. Cooperative relaying is introduced to extend the coverage and improve energy efficiency of OFDM-SNM [23]. Joint mapping technique is applied to OFDM-SNM to allow a constant length of information bits transmitted by OFDMSNM blocks [24]. Smart detector based on cascaded neural computing is designed to realize low-computational detection of OFDM-SNM blocks [25]. The concept of number modulation has also been transferred to the spatial domain to invent antenna subcarrier modulation (ASM) for multipleinput multiple-output (MIMO) systems in [26] and further developed with adaptive antenna selection and a power-domain non-orthogonal multi-access (NOMA) scheme in [27] and [28], respectively.

Although there have been a number of papers analyzing the error performance, outage performance, and spectral efficiency of OFDM-SNM, its performance gain over plain OFDM has not been rigorously studied from the information-theoretic perspective. Unlike OFDM-IM that has been analyzed in terms of achievable rate, mutual information, and channel capacity [29]-[31], only phenomenological research of OFDM-SNM has hitherto been carried out without a solid supporting pillar from information theory. So far, the performance limit of 
OFDM-SNM remains undiscovered, let alone the quantitative study of the performance gain brought by the novel number dimension in conjunction with conventional amplitude and phase dimensions.

To bridge this cognitional gap, we in this paper analyze the mutual information and channel capacity of OFDM-SNM. Since the analysis of mutual information and channel capacity are rather complex when the assignments of SAPs and constellation symbols are coupled, we adopt a set of powerful mathematical tools and assumptions to facilitate our analysis in this paper, including a successive padding procedure for establishing an explicit bijective mapping relation, the log sum inequality, and the Kullback-Leibler divergence. We also propose and apply several iterative algorithms jointly to search achievable solutions in an efficient manner. The analytical methodologies and derivations can be directly applied to analyze other cognate sparisity modulation schemes and can also reflect the information-theoretic nature of the number dimension in other domains. Specifically, the technical contributions of this paper are summarized as follows:

- We formulate and analyze the mutual information and channel capacity of OFDM-SNM.

- We derive an upper bound on the mutual information of OFDM-SNM in closed form by using the log sum inequality.

- We propose an easy-to-implement algorithm to approach the optimal SAP utilization probabilities (PUPs) in an iterative manner.

- We propose a Huffman coding based scheme to determine the achievable PUPs for practical implementation.

- We prove that the Huffman coding based scheme is capable of providing the best fit between the optimal PUPs and the achievable PUPs in the sense of Kullback-Leibler divergence.

- We verify the information-theoretic performance analysis in this paper by numerical simulations.

The rest of this paper is organized as follows. In Section II, we present the system model of OFDM-SNM. Based on this system model, we formulate and analyze the mutual information of OFDM-SNM in Section III. With the formulation of mutual information and a set of assumptions, we carry out the information-theoretic analysis of the channel capacity of OFDM-SNM in Section IV. Numerical results are presented and discussed in Section $\mathrm{V}$ to verify our information-theoretic analysis. Finally, the paper is concluded with several future research directions in Section VI.

Notation: $H(\cdot)$ and $I(\cdot ; \cdot)$ represent the entropy and mutual information; $D(\cdot \| \cdot)$ is the Kullback-Leibler divergence (a.k.a. the relative entropy) measuring the distance from a certain probability distribution to the reference probability distribution; $(\cdot)^{T}$ and $(\cdot)^{H}$ denote transpose and Hermitian transpose of vector/matrix, respectively; $\mathcal{C N}(\cdot, \cdot)$ represents the complex Gaussian distribution with the mean and variance enclosed; $(., \cdot, \ldots,$.$) is the multinomial coefficient, and \lfloor\cdot\rfloor$ is the floor function returning the nearest integer less than or equal to the enclosed; $\mathbb{E}\{\cdot\}$ gives the expectation of the random vector/matrix enclosed, and $\mathbb{P}\{\cdot\}$ denotes the probability of

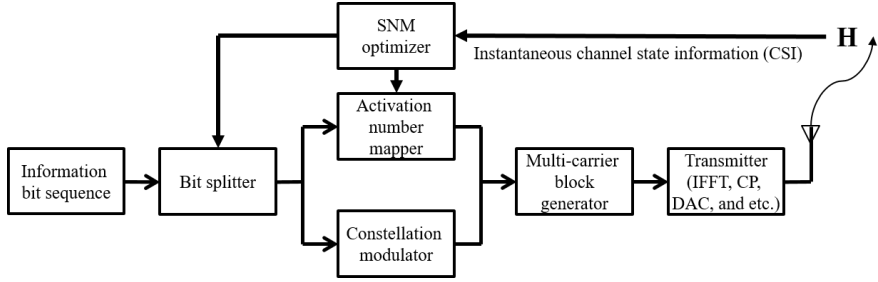

Fig. 1: OFDM-SNM transmitter structure with a single OFDM block.

the random vector/matrix enclosed.

\section{SySTEM MOdEL}

Without loss of generality, we consider an OFDM-SNM system with a single block of $N_{\mathrm{S}}$ subcarriers ${ }^{1}$, which form the full set of subcarriers $\mathcal{N}_{\mathrm{S}}=\left\{1,2, \ldots, N_{\mathrm{S}}\right\}$. Such an OFDMSNM system has a transmitter structure as shown in Fig. 1. According to this transmitter structure, the incoming bit sequence with length $p$ from the information source is first split into two parts with lengths $p_{\mathrm{A}}$ and $p_{\mathrm{C}}$ sent to the activation number mapper and the constellation modulator, respectively. In this paper, all bits are assumed to be independently distributed and equiprobable between ' 1 ' and ' 0 ', adhering to the principle of maximum entropy. Then, the activation number mapper can decide the number of active subcarriers $K_{\mathrm{A}}$ depending on the input $p_{\mathrm{A}}$-bit sequence. In parallel, the constellation modulator performs as the same as for plain OFDM with $K_{\mathrm{A}}$ subcarriers and generate the corresponding constellation symbols with certain amplitudes and phases based on the $p_{\mathrm{C}}$-bit sequence. Note that, to fully exploit the system-level design freedom and advantageous features of OFDM-SNM, $K_{\mathrm{A}} \geq 1$ is a varying number and has a bijective mapping relation with the input $p_{\mathrm{A}^{-}}$ bit sequence. As a direct consequence of the varying number of active subcarriers, the number of carried constellation symbols generated by the constellation modulator is also varying for each transmission attempt. This indicates that OFDM-SNM is a modulation scheme transmitting a varying length of bit sequence, which leads $p$ to a non-fixed value. The number of active subcarriers and the same number of constellation symbols are sent to the multi-carrier block generator and the following signal processing units to yield an independent transmission block with $N_{\mathrm{S}}$ elements, including $K_{\mathrm{A}}$ effective elements and $K_{\mathrm{I}}=N_{\mathrm{S}}-K_{\mathrm{A}}$ nulls. The following signal processing units include inverse fast Fourier transform (IFFT), cyclic prefix (CP) appending, digital-to-analog conversion (DAC), and up-conversion [33].

Different from the canonical OFDM-SNM scheme without any adaptation mechanism [18], we introduce an SNM optimizer in the OFDM-SNM transmitter structure that is able to adapt the codebook by instantaneous channel state information

\footnotetext{
${ }^{1}$ As detailed in [18] and [32], a near-optimal energy-based detection scheme can be employed for OFDM-SNM, which allows a relative large number of subcarriers in a single OFDM block. Therefore, the initial splitting of a total number of subcarriers into sub-blocks becomes unnecessary in practical application scenarios and is omitted in this paper for simplicity.
} 
(CSI) for maximizing the system transmission capability ${ }^{2}$. As a consequence of the adaptive codebook, the SNM optimizer also has an impact on the mapping relation between $p_{\mathrm{A}}$ input bits and the modulated SAP. In particular, for each $K_{\mathrm{A}}$, we assume that a certain SAP

$$
\mathbf{x}_{\mathrm{SNM}}\left(K_{\mathrm{A}}\right)=\left[\phi_{1}, \phi_{2}, \ldots, \phi_{N_{\mathrm{S}}}\right]^{T}
$$

is selected by a designed channel-dependent criterion from the SAP set denoted as $\mathcal{X}_{\mathrm{SNM}}\left(K_{\mathrm{A}}\right)$, where $\phi_{n}=1$ indicates that the $n$th subcarrier is active, and $\phi_{n}=0$ indicates that the corresponding subcarrier is inactive, $\forall n \in \mathcal{N}_{\mathrm{S}}$. The channeldependent SAP selection criterion for a given $K_{\mathrm{A}}$ is in nature a subcarrier assignment scheme relying on instantaneous CSI, which is detailed in [19]. In brief, the set of $K_{\mathrm{A}}$ active subcarriers denoted as $\mathcal{N}_{\mathrm{S}}\left(K_{\mathrm{A}}\right)$ is determined by ${ }^{3}$

$$
\mathcal{N}_{\mathrm{S}}\left(K_{\mathrm{A}}\right)=\underset{\aleph \subset \mathcal{N},|\aleph|=K_{\mathrm{A}}}{\arg \max }\left\{\sum_{n \in \aleph}\left|h_{n}\right|^{2}\right\},
$$

where $h_{n}$ is the instantaneous channel coefficient of the $n$th subcarrier, and the cardinality of $\mathcal{N}_{\mathrm{S}}\left(K_{\mathrm{A}}\right)$ is $\left|\mathcal{N}_{\mathrm{S}}\left(K_{\mathrm{A}}\right)\right|=K_{\mathrm{A}}$.

Obviously the cardinality of $\mathcal{X}_{\mathrm{SNM}}\left(K_{\mathrm{A}}\right)$ is $\left|\mathcal{X}_{\mathrm{SNM}}\left(K_{\mathrm{A}}\right)\right|=$ $\left(\begin{array}{l}N_{\mathrm{S}} \\ K_{\mathrm{A}}\end{array}\right)$. For each CSI updating round, $N_{\mathrm{T}}=2^{\left\lfloor\log _{2}\left(N_{\mathrm{S}}\right)\right\rfloor} \leq N_{\mathrm{S}}$ times of SAP selection are carried out to form an optimized codebook, which consists of

$$
\sum_{K_{\mathrm{A}} \in \mathcal{N}_{\mathrm{T}}}\left(\begin{array}{l}
N_{\mathrm{S}} \\
K_{\mathrm{A}}
\end{array}\right)=2^{N_{\mathrm{S}}}-1-\Delta\left(N_{\mathrm{S}}, N_{\mathrm{T}}\right)
$$

selection processes, where

$$
\Delta\left(N_{\mathrm{S}}, N_{\mathrm{T}}\right)=\frac{N_{\mathrm{S}} \Gamma\left(N_{\mathrm{S}}\right)_{2} \tilde{F}_{1}\left(1,-N_{\mathrm{S}}+N_{\mathrm{T}}+1 ; N_{\mathrm{T}}+2 ;-1\right)}{\Gamma\left(N_{\mathrm{S}}-N_{\mathrm{T}}\right)}
$$

signifies the design freedom when $N_{\mathrm{T}}<N_{\mathrm{S}}$ and equals zero when ${ }^{4} N_{\mathrm{T}}=N_{\mathrm{S}} ;{ }_{2} \tilde{F}_{1}(\cdot, \cdot ; \cdot ; \cdot)$ is the regularized hypergeometric function, and $\Gamma(\cdot)$ is the gamma function.

Once $\mathrm{x}_{\mathrm{SNM}}\left(K_{\mathrm{A}}\right)$ is given, $K_{\mathrm{A}}$ constellation symbols generated by the constellation modulator will be carried on the $K_{\mathrm{A}}$ active subcarriers. For $M$-ary amplitude-phase modulation (APM) with a symbol set $\mathcal{M}$, e.g., $M$-ary phase shift keying

\footnotetext{
${ }^{2}$ It should be noted that instantaneous CSI is not a necessity of performing OFDM-SNM but more like a bonus that produces a coding gain by activating subcarriers with bias [19]. Without the access to instantaneous CSI, OFDMSNM can still be carried out by activating subcarriers in sequence [18]. Considering the convenience for estimating OFDM channels in quasi-static propagation environments for the applications in the IoT and MTC networks [34], it is reasonable to assume that instantaneous CSI is perfectly accessible.

${ }^{3}$ Given $K_{\mathrm{A}}$, the designed assignment criterion is equivalent to identifying $K_{\mathrm{A}}$ subcarriers with the first, second and until the $K_{\mathrm{A}}$ th largest instantaneous channel power gains from $N_{\mathrm{S}}$ subcarriers. Considering a toy example when $N_{\mathrm{S}}=4$ and $\left[\left|h_{1}\right|^{2},\left|h_{2}\right|^{2},\left|h_{3}\right|^{2},\left|h_{4}\right|^{2}\right]=$ $[1.2672,0.4811,2.9746,0.9988]$, we have $\left|h_{3}\right|^{2}>\left|h_{1}\right|^{2}>\left|h_{4}\right|^{2}>\left|h_{2}\right|^{2}$ Then, the assignment criterion will produce: $\mathcal{N}_{\mathrm{S}}(1)=\{3\}, \mathcal{N}_{\mathrm{S}}(2)=\{1,3\}$, $\mathcal{N}_{\mathrm{S}}(3)=\{1,3,4\}$, and $\mathcal{N}_{\mathrm{S}}(4)=\{1,2,3,4\}$.

${ }^{4}$ In most modern OFDM systems where IFFT is performed, $N_{\mathrm{T}}=N_{\mathrm{S}}$ must be satisfied [33]. We present the above discussion without presuming $N_{\mathrm{T}}=N_{\mathrm{S}}$ to keep the generality of this work. For the rest of this paper, $N_{\mathrm{T}}=N_{\mathrm{S}}$ is implied, unless explicitly stated otherwise. Meanwhile, to avoid bring confusion, we still keep using both notations $N_{\mathrm{S}}$ and $N_{\mathrm{T}}$ for the total number of subcarriers and the maximum number of active subcarriers as well as the total number of legitimate SAPs, respectively.
}

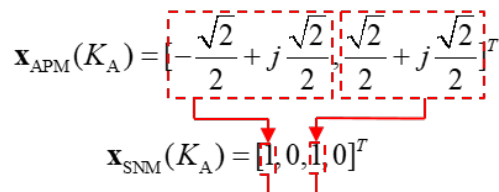

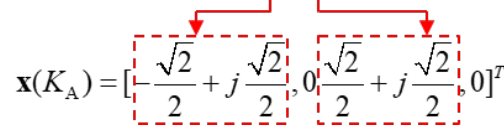

Fig. 2: An illustrative example of the successive padding procedure, given $N_{\mathrm{S}}=4, K_{\mathrm{A}}=2$, and $M=4(\mathrm{QPSK})$.

( $M$-PSK) and $M$-ary quadrature amplitude modulation ( $M$ QAM), there are $M$ options available for each symbol decided by a $\log _{2}(M)$-bit segment of the $p_{\mathrm{C}}$-bit input sequence to the constellation modulator ${ }^{5}$. Assuming Gray coding is implemented to map the bits to a symbol, we have $p_{\mathrm{C}}=$ $K_{\mathrm{A}} \log _{2}(M)$. Overall, a certain symbol combination pattern (SCP)

$$
\mathbf{x}_{\mathrm{APM}}\left(K_{\mathrm{A}}\right)=\left[s_{1}, s_{2}, \ldots, s_{K_{\mathrm{A}}}\right]^{T}
$$

is determined by the $p_{\mathrm{C}}$-bit input sequence of the constellation modulator, where $s_{n}$ is a normalized $M$-ary complex symbol with the property $\mathbb{E}\left\{\left(s_{n}\right)^{H} s_{n}\right\}=1$.

With both SAP $\mathbf{x}_{\mathrm{SNM}}\left(K_{\mathrm{A}}\right)$ and $\mathrm{SCP} \mathbf{x}_{\mathrm{APM}}\left(K_{\mathrm{A}}\right)$, we can construct the multi-carrier transmission block in a bijective manner as

$$
\mathbf{x}\left(K_{\mathrm{A}}\right)=\left[x_{1}, x_{2}, \ldots, x_{N_{\mathrm{S}}}\right]^{T}
$$

for OFDM-SNM by a successive padding procedure:

$$
x_{n}=\left\{\begin{array}{ll}
s_{\eta(n)}, & \text { if } \phi_{n}=1 \\
0, & \text { if } \phi_{n}=0
\end{array},\right.
$$

where $\eta(n)$ maps the index of the active subcarrier, i.e., $n$, to the index of the padded symbol in $\mathrm{x}_{\mathrm{APM}}\left(K_{\mathrm{A}}\right)$ in a successive order. This successive padding procedure can be pictorially illustrated in Fig. 2 for clarity. Because $\mathbb{E}\left\{\left(s_{n}\right)^{H} s_{n}\right\}=1$, we can easily have $\mathbb{E}\left\{\left(\mathbf{x}\left(K_{\mathrm{A}}\right)\right)^{H} \mathbf{x}\left(K_{\mathrm{A}}\right)\right\}=K_{\mathrm{A}}$.

Assuming a given amount of transmit power $P_{\mathrm{T}}$ is uniformly allocated to $K_{\mathrm{A}}$ subcarriers, the received multi-carrier block after CP removal and FFT at the receiving side can be expressed as

$$
\mathbf{y}\left(K_{\mathrm{A}}\right)=\left[y_{1}, y_{2}, \ldots, y_{N_{\mathrm{S}}}\right]^{T}=\sqrt{\frac{P_{\mathrm{T}}}{K_{\mathrm{A}}}} \mathbf{H} \mathbf{x}\left(K_{\mathrm{A}}\right)+\mathbf{w},
$$

where $\mathbf{H}=\operatorname{diag}\left\{h_{1}, h_{2}, \ldots, h_{N_{\mathrm{S}}}\right\}$ is an $N_{\mathrm{S}} \times N_{\mathrm{S}}$ diagonal channel coefficient matrix, and $\mathbf{w}=\left[w_{1}, w_{2}, \ldots, w_{N_{\mathrm{S}}}\right]^{T}$ is the an $N_{\mathrm{S}} \times 1$ noise vector. For simplicity, we assume that the noise is an independent frequency-domain additive white Gaussian noise (AWGN) abiding $\mathcal{C N}\left(0, B_{n} N_{0}\right)$, where $B_{n}$ is the bandwidth of the $n$th subcarrier, and $N_{0}$ is the power spectral density (PSD) of noise. Viewing the complex random variables as a pair of real and imaginary random components, we can treat $h_{n}$ and $w_{n}$ as two-dimensional random vectors. Because for most OFDM systems, we have $B=B_{1}=B_{2}=$

\footnotetext{
${ }^{5}$ Without loss of generality, the base of the logarithm is set to two for all information measures in this paper. Therefore, the resulting units of all information measures in this paper are bits.
} 


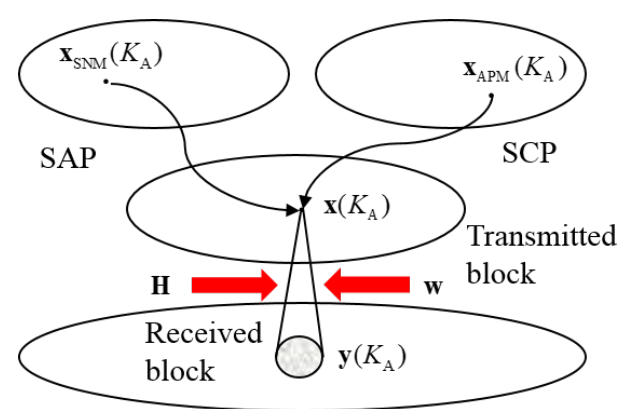

Fig. 3: A diagrammatic representation of the mapping relation among $\mathbf{x}_{\mathrm{SNM}}\left(K_{\mathrm{A}}\right), \mathbf{x}_{\mathrm{APM}}\left(K_{\mathrm{A}}\right), \mathbf{x}\left(K_{\mathrm{A}}\right)$, and $\mathbf{y}\left(K_{\mathrm{A}}\right)$. The circles represent the legitimate sets of the corresponding random vectors.

$\cdots=B_{N_{\mathrm{S}}}$ [35], the probability density function (PDF) of $w_{n}$ can be determined by [33]

$$
f_{w}\left(w_{n}\right)=\frac{1}{\pi B N_{0}} \exp \left(-\frac{\left|w_{n}\right|^{2}}{B N_{0}}\right) .
$$

From (7) and (8), we can also have the entry-wise relation between the transmitted and received multi-carrier blocks as

$$
y_{n}=\sqrt{\frac{P_{\mathrm{T}}}{K_{\mathrm{A}}}} h_{n} x_{n}+w_{n}= \begin{cases}\sqrt{\frac{P_{\mathrm{T}}}{K_{\mathrm{A}}}} h_{n} s_{\eta(n)}+w_{n}, & \text { if } \phi_{n}=1 \\ w_{n}, & \text { if } \phi_{n}=0\end{cases}
$$

Based on the descriptions above, the mapping relation among $\mathbf{x}_{\mathrm{SNM}}\left(K_{\mathrm{A}}\right), \mathbf{x}_{\mathrm{APM}}\left(K_{\mathrm{A}}\right), \mathbf{x}\left(K_{\mathrm{A}}\right)$, and $\mathbf{y}\left(K_{\mathrm{A}}\right)$ can be diagrammatically shown in Fig. 3. Accordingly, the receiver can employ a certain detection scheme, e.g., maximum likelihood (ML) detection, log-likelihood ratio (LLR) detection, and energy-based detection, to estimate and reconstruct $\mathrm{x}\left(K_{\mathrm{A}}\right)$ by $\mathbf{y}\left(K_{\mathrm{A}}\right)$ [18], [22], [36]. Subsequently, both $\mathbf{x}_{\mathrm{SNM}}\left(K_{\mathrm{A}}\right)$ and $\mathbf{x}_{\mathrm{APM}}\left(K_{\mathrm{A}}\right)$ can be obtained from $\mathbf{x}\left(K_{\mathrm{A}}\right)$ through the bijective relation resulted by the successive padding procedure. Finally, the original $p$-bit sequence is thereby retrieved by the OFDMSNM demodulation afterwards.

\section{Formulation AND ANAlysis of Mutual INFORMATION}

As shown in Fig. 3, because the successive padding procedure establishes a bijective mapping relation between $\mathbf{x}_{\mathrm{SNM}}\left(K_{\mathrm{A}}\right), \mathbf{x}_{\mathrm{APM}}\left(K_{\mathrm{A}}\right)$, and $\mathbf{x}\left(K_{\mathrm{A}}\right)$, it is obvious that $H\left(\mathbf{x}\left(K_{\mathrm{A}}\right)\right)=H\left(\mathbf{x}_{\mathrm{SNM}}\left(K_{\mathrm{A}}\right), \mathbf{x}_{\mathrm{APM}}\left(K_{\mathrm{A}}\right)\right)$. Hence, we can reduce the mutual information of OFDM-SNM to be

$$
I\left(\mathbf{x}\left(K_{\mathrm{A}}\right) ; \mathbf{y}\left(K_{\mathrm{A}}\right)\right)=I\left(\mathbf{x}_{\mathrm{SNM}}\left(K_{\mathrm{A}}\right), \mathbf{x}_{\mathrm{APM}}\left(K_{\mathrm{A}}\right) ; \mathbf{y}\left(K_{\mathrm{A}}\right)\right) .
$$

The mutual information given above can be further reduced by the chain rule for mutual information to be [37]

$$
\begin{aligned}
I\left(\mathbf{x}_{\mathrm{SNM}}\left(K_{\mathrm{A}}\right), \mathbf{x}_{\mathrm{APM}}\left(K_{\mathrm{A}}\right) ; \mathbf{y}\left(K_{\mathrm{A}}\right)\right) \\
=I\left(\mathbf{x}_{\mathrm{APM}}\left(K_{\mathrm{A}}\right) ; \mathbf{y}\left(K_{\mathrm{A}}\right) \mid \mathbf{x}_{\mathrm{SNM}}\left(K_{\mathrm{A}}\right)\right) \\
\quad+I\left(\mathbf{x}_{\mathrm{SNM}}\left(K_{\mathrm{A}}\right) ; \mathbf{y}\left(K_{\mathrm{A}}\right)\right) .
\end{aligned}
$$

Denote the probability that $K_{\mathrm{A}}$ subcarriers are activated as $\mathrm{P}\left(\mathbf{x}_{\mathrm{SNM}}\left(K_{\mathrm{A}}\right)\right)$. According to the basic definition of conditional mutual information, we can expand the conditional mutual information between $\mathbf{x}_{\mathrm{APM}}\left(K_{\mathrm{A}}\right)$ and $\mathbf{y}\left(K_{\mathrm{A}}\right)$ given $\mathbf{x}_{\mathrm{SNM}}\left(K_{\mathrm{A}}\right)$ in (13), where (a) is valid upon the assumptions that all incoming bit sequences are equiprobable and Gray coding is implemented. As a result, once an SAP with $k$ active subcarriers is given, all $M^{k}$ SCPs are chosen equally with a uniform probability $1 / M^{k}$. This conditional mutual information measures how much information regarding the SCP can be obtained by estimating received multi-carrier block once the SAP has already been correctly estimated.

In (13), $f_{\mathbf{y}}\left(\mathbf{y}(k) \mid \mathbf{x}_{\mathrm{SNM}}\left(K_{\mathrm{A}}\right)=\mathbf{x}_{\mathrm{SNM}}(k), \mathbf{x}_{\mathrm{APM}}\left(K_{\mathrm{A}}\right)=\right.$ $\left.\mathbf{x}_{\mathrm{APM}}(k)\right)$ is the conditional PDF of $\mathbf{y}(k)$ given $\mathbf{x}_{\mathrm{SNM}}\left(K_{\mathrm{A}}\right)=$ $\mathbf{x}_{\mathrm{SNM}}(k)$ and $\mathbf{x}_{\mathrm{APM}}\left(K_{\mathrm{A}}\right)=\mathbf{x}_{\mathrm{APM}}(k)$ with $k$ active subcarriers. Because of the independent nature of OFDM systems over multiple subcarriers, we can first discuss the entry-wise conditional distribution of each $y_{n}$ given the SAP and SCP and then derive the joint conditional distribution of $\mathbf{y}(k)$.

In (13), $f_{\mathbf{y}}\left(\mathbf{y}(k) \mid \mathbf{x}_{\mathrm{SNM}}\left(K_{\mathrm{A}}\right)=\mathbf{x}_{\mathrm{SNM}}(k)\right)$ is the conditional PDF of $\mathbf{y}(k)$ solely conditioned on $\mathbf{x}_{\mathrm{SNM}}\left(K_{\mathrm{A}}\right)=\mathbf{x}_{\mathrm{SNM}}(k)$. By the basics of probability theory, we tentatively regard $h_{n}$ as a known and stationary factor and derive the conditional PDF of $y_{n}$ as

$$
f_{\mathrm{A} / \mathrm{I}}^{k}\left(y_{n}\right)=\frac{1}{\pi \varrho_{n}^{k}} \exp \left(-\frac{\left|y_{n}\right|^{2}}{\varrho_{n}^{k}}\right),
$$

where

$$
\varrho_{n}^{k}=\left\{\begin{array}{ll}
B N_{0}\left(\frac{P_{\mathrm{T}}\left|h_{n}\right|^{2}}{k B N_{0}}+1\right), & \text { if } n \in \mathcal{N}_{\mathrm{S}}(k) \\
B N_{0} & \text { if } n \notin \mathcal{N}_{\mathrm{S}}(k)
\end{array} .\right.
$$

Therefore, the joint distribution of $\mathbf{y}(k)$ solely conditioned on SAP can be characterized by its joint conditional PDF given by

$$
\begin{array}{r}
f_{\mathbf{y}}\left(\mathbf{y}(k) \mid \mathbf{x}_{\mathrm{SNM}}\left(K_{\mathrm{A}}\right)=\mathbf{x}_{\mathrm{SNM}}(k)\right)=\prod_{n=1}^{N_{\mathrm{S}}} f_{\mathrm{A} / \mathrm{I}}^{k}\left(y_{n}\right) \\
=\left(\prod_{n \in \mathcal{N}_{\mathrm{S}}(k)} f_{\mathrm{A}}^{k}\left(y_{n}\right)\right)\left(\prod_{n \notin \mathcal{N}_{\mathrm{S}}(k)} f_{\mathrm{I}}\left(y_{n}\right)\right) .
\end{array}
$$

In addition, from the basic definition of mutual information, we can similarly expand the mutual information between $\mathbf{x}_{\mathrm{SNM}}\left(K_{\mathrm{A}}\right)$ and $\mathbf{y}\left(K_{\mathrm{A}}\right)$ as

$$
\begin{aligned}
& I\left(\mathbf{x}_{\mathrm{SNM}}\left(K_{\mathrm{A}}\right) ; \mathbf{y}\left(K_{\mathrm{A}}\right)\right)=\sum_{k=1}^{N_{\mathrm{T}}} \mathrm{P}\left(\mathbf{x}_{\mathrm{SNM}}(k)\right) \\
& \quad \times \int_{\mathbf{y}(k)} f_{\mathbf{y}}\left(\mathbf{y}(k) \mid \mathbf{x}_{\mathrm{SNM}}\left(K_{\mathrm{A}}\right)=\mathbf{x}_{\mathrm{SNM}}(k)\right) \\
& \quad \times \log _{2}\left(\frac{f_{\mathbf{y}}\left(\mathbf{y}(k) \mid \mathbf{x}_{\mathrm{SNM}}\left(K_{\mathrm{A}}\right)=\mathbf{x}_{\mathrm{SNM}}(k)\right)}{f_{\mathbf{y}}(\mathbf{y}(k))}\right) \mathrm{d} \mathbf{y}(k),
\end{aligned}
$$

where $f_{\mathbf{y}}(\mathbf{y}(k))$ is the a priori PDF of $\mathbf{y}(k)$ without any prior knowledge of $\mathbf{x}_{\mathrm{SNM}}\left(K_{\mathrm{A}}\right)$ and $\mathbf{x}_{\mathrm{APM}}\left(K_{\mathrm{A}}\right)$. We can obtain the a priori PDF of $\mathbf{y}(k)$ by averaging $f_{\mathbf{y}}\left(\mathbf{y}(k) \mid \mathbf{x}_{\mathrm{SNM}}\left(K_{\mathrm{A}}\right)=\right.$ $\left.\mathbf{x}_{\mathrm{SNM}}(k)\right)$ over $\mathbf{x}_{\mathrm{SNM}}\left(K_{\mathrm{A}}\right)=\mathbf{x}_{\mathrm{SNM}}(k)$ as

$f_{\mathbf{y}}(\mathbf{y}(k))=\sum_{i=1}^{N_{\mathrm{T}}} \mathrm{P}\left(\mathbf{x}_{\mathrm{SNM}}(i)\right) f_{\mathbf{y}}\left(\mathbf{y}(k) \mid \mathbf{x}_{\mathrm{SNM}}\left(K_{\mathrm{A}}\right)=\mathbf{x}_{\mathrm{SNM}}(i)\right)$.

As such, the mutual information $I\left(\mathbf{x}_{\mathrm{SNM}}\left(K_{\mathrm{A}}\right) ; \mathbf{y}\left(K_{\mathrm{A}}\right)\right)$ quantifies how much information regarding $\mathrm{x}_{\mathrm{SNM}}\left(K_{\mathrm{A}}\right)$ one can obtain by estimating $\mathbf{y}\left(K_{\mathrm{A}}\right)$. 


$$
\begin{aligned}
& I\left(\mathbf{x}_{\mathrm{APM}}\left(K_{\mathrm{A}}\right) ; \mathbf{y}\left(K_{\mathrm{A}}\right) \mid \mathbf{x}_{\mathrm{SNM}}\left(K_{\mathrm{A}}\right)\right)= \sum_{k=1}^{N_{\mathrm{T}}} \mathrm{P}\left(\mathbf{x}_{\mathrm{SNM}}(k)\right) I\left(\mathbf{x}_{\mathrm{APM}}\left(K_{\mathrm{A}}\right) ; \mathbf{y}\left(K_{\mathrm{A}}\right) \mid \mathbf{x}_{\mathrm{SNM}}\left(K_{\mathrm{A}}\right)=\mathbf{x}_{\mathrm{SNM}}(k)\right) \\
& \stackrel{(\mathrm{a})}{=} \sum_{k=1}^{N_{\mathrm{T}}} \mathrm{P}_{\mathbf{x}}\left(\mathbf{x}_{\mathrm{SNM}}(k)\right) \sum_{m=1}^{M^{k}} \frac{1}{M^{k}} \int_{\mathbf{y}(k)} f_{\mathbf{y}}\left(\mathbf{y}(k) \mid \mathbf{x}_{\mathrm{SNM}}\left(K_{\mathrm{A}}\right)=\mathbf{x}_{\mathrm{SNM}}(k), \mathbf{x}_{\mathrm{APM}}\left(K_{\mathrm{A}}\right)=\mathbf{x}_{\mathrm{APM}}(k)\right) \\
& \times \log _{2}\left(\frac{f_{\mathbf{y}}\left(\mathbf{y}(k) \mid \mathbf{x}_{\mathrm{SNM}}\left(K_{\mathrm{A}}\right)=\mathbf{x}_{\mathrm{SNM}}(k), \mathbf{x}_{\mathrm{APM}}\left(K_{\mathrm{A}}\right)=\mathbf{x}_{\mathrm{APM}}(k)\right)}{f_{\mathbf{y}}\left(\mathbf{y}(k) \mid \mathbf{x}_{\mathrm{SNM}}\left(K_{\mathrm{A}}\right)=\mathbf{x}_{\mathrm{SNM}}(k)\right)}\right) \mathrm{d} \mathbf{y}(k)
\end{aligned}
$$

\section{INFORMATION-THEORETIC ANALYSIS}

\section{A. Problem Formulation and Analysis}

The formulation and analysis of mutual information for OFDM-SNM directly lead to the formulation of channel capacity upon the stationary channel settings characterized by H [33]:

$$
\begin{aligned}
C(\mathbf{H})=\max _{\mathbf{P}}\{ & \left.I\left(\mathbf{x}_{\mathrm{SNM}}\left(K_{\mathrm{A}}\right), \mathbf{x}_{\mathrm{APM}}\left(K_{\mathrm{A}}\right) ; \mathbf{y}\left(K_{\mathrm{A}}\right)\right)\right\} \\
=\max _{\mathbf{P}}\{ & I\left(\mathbf{x}_{\mathrm{APM}}\left(K_{\mathrm{A}}\right) ; \mathbf{y}\left(K_{\mathrm{A}}\right) \mid \mathbf{x}_{\mathrm{SNM}}\left(K_{\mathrm{A}}\right)\right) \\
+ & \left.I\left(\mathbf{x}_{\mathrm{SNM}}\left(K_{\mathrm{A}}\right) ; \mathbf{y}\left(K_{\mathrm{A}}\right)\right)\right\}
\end{aligned}
$$

where $\mathbf{P}=\left[\mathrm{P}\left(\mathbf{x}_{\mathrm{SNM}}(1)\right), \mathrm{P}\left(\mathbf{x}_{\mathrm{SNM}}(2)\right), \ldots, \mathrm{P}\left(\mathbf{x}_{\mathrm{SNM}}\left(N_{\mathrm{T}}\right)\right)\right]^{T}$ is the PUP vector of $N_{\mathrm{T}}$ SAPs. It is evident that $\sum_{k=1}^{N_{\mathrm{T}}} \mathrm{P}\left(\mathbf{x}_{\mathrm{SNM}}(k)\right)=1$ and $^{6} 0 \leq \mathrm{P}\left(\mathbf{x}_{\mathrm{SNM}}(k)\right) \leq 1$.

In theory, we aim to find the optimal PUP vector $\mathbf{P}^{*}$ so as to achieve the maximum mutual information, i.e., channel capacity. The searching problem with regard to $\mathbf{P}^{*}$ is formulated by

$$
\begin{aligned}
\mathbf{P}^{*}=\left[\mathrm{P}^{*}\left(\mathbf{x}_{\mathrm{SNM}}(1)\right), \mathrm{P}^{*}\left(\mathbf{x}_{\mathrm{SNM}}(2)\right), \ldots, \mathrm{P}^{*}\left(\mathbf{x}_{\mathrm{SNM}}\left(N_{\mathrm{T}}\right)\right)\right]^{T} \\
=\underset{\mathbf{P}}{\arg \max }\left\{I\left(\mathbf{x}_{\mathrm{SNM}}\left(K_{\mathrm{A}}\right), \mathbf{x}_{\mathrm{APM}}\left(K_{\mathrm{A}}\right) ; \mathbf{y}\left(K_{\mathrm{A}}\right)\right)\right\} \\
\text { s.t. } \sum_{k=1}^{N_{\mathrm{T}}} \mathrm{P}\left(\mathbf{x}_{\mathrm{SNM}}(k)\right)=1, \\
\quad 0 \leq \mathrm{P}\left(\mathbf{x}_{\mathrm{SNM}}(k)\right) \leq 1, \forall 1 \leq k \leq N_{\mathrm{T}} .
\end{aligned}
$$

First, let us take a close look at $I\left(\mathbf{x}_{\mathrm{APM}}\left(K_{\mathrm{A}}\right) ; \mathbf{y}\left(K_{\mathrm{A}}\right) \mid \mathbf{x}_{\mathrm{SNM}}\left(K_{\mathrm{A}}\right)\right)$. If an $\mathrm{SAP}$ is given and fixed, this conditional mutual information can be analyzed as for a canonical OFDM with $K_{\mathrm{A}}$ parallel channels [38]. Therefore, this conditional term will reach its maximum when $\mathrm{x}_{\mathrm{APM}}\left(K_{\mathrm{A}}\right)$ is a vector with $K_{\mathrm{A}}$ power constrained

\footnotetext{
${ }^{6} \mathrm{Here}$, we intent to stipulate a closed set for the activation probability $\mathrm{P}\left(\mathbf{x}_{\mathrm{SNM}}(k)\right)$ for an arbitrary $\mathrm{SAP} \mathbf{x}_{\mathrm{SNM}}(k)$, implying that abandoning a subset of SAPs is allowed during the optimization. In an extreme case where $\mathrm{P}\left(\mathbf{x}_{\mathrm{SNM}}(1)\right)=\mathrm{P}\left(\mathbf{x}_{\mathrm{SNM}}(2)\right)=\cdots=\mathrm{P}\left(\mathbf{x}_{\mathrm{SNM}}\left(N_{\mathrm{T}}-\right.\right.$ $1))=0$ and $\mathrm{P}\left(\mathbf{x}_{\mathrm{SNM}}\left(N_{\mathrm{T}}\right)\right)=1$, OFDM-SNM reduces to plain OFDM utilizing $N_{\mathrm{T}}$ available subcarriers from the optimized set $\mathcal{N}_{\mathrm{S}}\left(N_{\mathrm{T}}\right)=$ $\arg \max \quad\left\{\sum_{n \in \mathcal{N}}\left|h_{n}\right|^{2}\right\}$ formed by the subcarrier assignment. This $\aleph \subset \mathcal{N}_{\mathrm{S}},|\aleph|=N_{\mathrm{T}}$

closed stipulation is able to release the underlying presumption of OFDMSNM that all $N_{\mathrm{T}}$ SAPs must be used and therefore scrutinize the necessity of OFDM-SNM.
}

continuous Gaussian distributed inputs and $K_{\mathrm{I}}$ nulls [33]. As a result, we can derive the following inequality:

$$
\begin{aligned}
& I\left(\mathbf{x}_{\mathrm{APM}}\left(K_{\mathrm{A}}\right) ; \mathbf{y}\left(K_{\mathrm{A}}\right) \mid \mathbf{x}_{\mathrm{SNM}}\left(K_{\mathrm{A}}\right)\right) \\
& <\bar{I}\left(\mathbf{x}_{\mathrm{APM}}\left(K_{\mathrm{A}}\right) ; \mathbf{y}\left(K_{\mathrm{A}}\right) \mid \mathbf{x}_{\mathrm{SNM}}\left(K_{\mathrm{A}}\right)\right) \\
& =\sum_{k=1}^{N_{\mathrm{T}}} \mathrm{P}\left(\mathbf{x}_{\mathrm{SNM}}(k)\right) \sum_{n \in \mathcal{N}_{\mathrm{S}}(k)} B \log _{2}\left(1+\frac{P_{\mathrm{T}}\left|h_{n}\right|^{2}}{k B N_{0}}\right) \text {. }
\end{aligned}
$$

Therefore, we can approximate the conditional mutual information given a certain SAP $I\left(\mathbf{x}_{\mathrm{APM}}\left(K_{\mathrm{A}}\right) ; \mathbf{y}\left(K_{\mathrm{A}}\right) \mid \mathbf{x}_{\mathrm{SNM}}\left(K_{\mathrm{A}}\right)\right)$ by its upper bound $\bar{I}\left(\mathbf{x}_{\mathrm{APM}}\left(K_{\mathrm{A}}\right) ; \mathbf{y}\left(K_{\mathrm{A}}\right) \mid \mathbf{x}_{\mathrm{SNM}}\left(K_{\mathrm{A}}\right)\right)$, which is a linear combination of $\mathbf{P}$ as shown in (21).

Second, referring to (17), we can resort to Bayes' theorem to have the following relation

$$
\frac{f_{\mathbf{y}}\left(\mathbf{y}(k) \mid \mathbf{x}_{\mathrm{SNM}}\left(K_{\mathrm{A}}\right)=\mathbf{x}_{\mathrm{SNM}}(k)\right)}{f_{\mathbf{y}}(\mathbf{y}(k))}=\frac{\mathrm{P}\left(\mathbf{x}_{\mathrm{SNM}}(k) \mid \mathbf{y}(k)\right)}{\mathrm{P}\left(\mathbf{x}_{\mathrm{SNM}}(k)\right)}
$$

where the a posteriori PUP $\mathrm{P}\left(\mathbf{x}_{\mathrm{SNM}}(k) \mid \mathbf{y}(k)\right)$ given the knowledge of $\mathbf{y}(k)$ can be derived by the formula of total probability as

$$
\begin{aligned}
& \mathrm{P}\left(\mathbf{x}_{\mathrm{SNM}}(k) \mid \mathbf{y}(k)\right) \\
& =\frac{\mathrm{P}\left(\mathbf{x}_{\mathrm{SNM}}(k)\right) f_{\mathbf{y}}\left(\mathbf{y}(k) \mid \mathbf{x}_{\mathrm{SNM}}\left(K_{\mathrm{A}}\right)=\mathbf{x}_{\mathrm{SNM}}(k)\right)}{\sum_{i=1}^{N_{\mathrm{T}}} \mathrm{P}\left(\mathbf{x}_{\mathrm{SNM}}(i)\right) f_{\mathbf{y}}\left(\mathbf{y}(i) \mid \mathbf{x}_{\mathrm{SNM}}\left(K_{\mathrm{A}}\right)=\mathbf{x}_{\mathrm{SNM}}(i)\right)} .
\end{aligned}
$$

Accordingly, we rewrite $I\left(\mathbf{x}_{\mathrm{SNM}}\left(K_{\mathrm{A}}\right) ; \mathbf{y}\left(K_{\mathrm{A}}\right)\right)$ as

$$
\begin{aligned}
& I\left(\mathbf{x}_{\mathrm{SNM}}\left(K_{\mathrm{A}}\right) ; \mathbf{y}\left(K_{\mathrm{A}}\right)\right)=\sum_{k=1}^{N_{\mathrm{T}}} \mathrm{P}\left(\mathbf{x}_{\mathrm{SNM}}(k)\right) \\
& \times \int_{\mathbf{y}(k)} f_{\mathbf{y}}\left(\mathbf{y}(k) \mid \mathbf{x}_{\mathrm{SNM}}\left(K_{\mathrm{A}}\right)=\mathbf{x}_{\mathrm{SNM}}(k)\right) \\
& \quad \times \log _{2}\left(\frac{\mathrm{P}\left(\mathbf{x}_{\mathrm{SNM}}(k) \mid \mathbf{y}(k)\right)}{\mathrm{P}\left(\mathbf{x}_{\mathrm{SNM}}(k)\right)}\right) \mathrm{d} \mathbf{y}(k) .
\end{aligned}
$$

Denote the state space of $\mathbf{P}$ as $\Theta(\mathbf{P})=[0,1]^{N_{\mathrm{T}} \times 1}$, which is obviously a convex set. Also, $I\left(\mathbf{x}_{\mathrm{SNM}}\left(K_{\mathrm{A}}\right) ; \mathbf{y}\left(K_{\mathrm{A}}\right)\right)$ given in the rewritten form can be proven to be a concave function of $^{7} \mathbf{P}$. Therefore, the objective function of the optimization problem (20) consisting of a linear combination of $\mathbf{P}$ and a concave function of $\mathbf{P}$ is also a concave function of $\mathbf{P}$. Hence, the Karush-Kuhn-Tucker (KKT) conditions are satisfied, and accordingly there must exist a single extremum

\footnotetext{
${ }^{7}$ Interested readers can refer to Appendix A for a rigorous proof.
} 
$\mathbf{P}^{*}$ that is the optimum and maximizes the objective function $I\left(\mathbf{x}_{\mathrm{SNM}}\left(K_{\mathrm{A}}\right), \mathbf{x}_{\mathrm{APM}}\left(K_{\mathrm{A}}\right) ; \mathbf{y}\left(K_{\mathrm{A}}\right)\right)$. The existence of the optimum $\mathbf{P}^{*}$ is unique and axiomatic. Interesting readers can find rigorous but tedious proofs in [39]. As a direct consequence of the concavity, there exists at least one iterative algorithm that can converge toward the maximum in a monotonically non-decreasing manner [40], [41].

From (21) and (24), we can form a systematic view of OFDM-SNM from the information-theoretic perspective. In information theory parlance, the channel resulted by OFDMSNM is in nature a hybrid channel merging the parallel channel model and the sum channel model with $N_{\mathrm{T}}$ paths via weights taken from $\mathbf{P}$.

\section{B. Iterative Algorithm to Approach the Optimum}

To fast converge toward the channel capacity and yield the optimal PUP vector, an iterative algorithm for optimal spatialdomain design has been proposed for spatial modulation (SM) in [42]. The core principle of this iterative algorithm has also been numerically verified to be effective for optimal frequency-domain design for OFDM-IM in [30]. In this subsection, we tailor this iterative algorithm for OFDM-SNM by considering the information brought by the subcarrier number dimension. It can be shown that the computational complexity of the tailored iterative algorithm is linearly proportional to the number of assigned subcarriers for OFDM-SNM, i.e., $N_{\mathrm{T}}$. This linear property makes it a suited algorithm for practical OFDM-SNM systems with a large $N_{\mathrm{T}}$, subject to volatile channel conditions and requiring real-time optimization. Specifically, the tailored iterative algorithm is a finite-step approach, and we detail these steps as follows.

Step 1: Initialize: Denote the the PUP of $\operatorname{SAP} \mathbf{x}_{\mathrm{SNM}}(k)$ yielded by the tailored iterative algorithm in the $\tau$ th iteration as $\mathrm{P}_{\langle\tau\rangle}\left(\mathbf{x}_{\mathrm{SNM}}(k)\right)$. For the first iteration, i.e., $\tau=1$, we initialize the PUPs for all $N_{\mathrm{T}}$ SAPs as

$$
\mathrm{P}_{\langle 1\rangle}\left(\mathbf{x}_{\mathrm{SNM}}(k)\right)=1 / N_{\mathrm{T}}, \forall k \in\left[1,2, \ldots, N_{\mathrm{T}}\right] .
$$

We organize all PUPs for each iteration by the vector $\mathbf{P}_{\langle\tau\rangle}=$ $\left[\mathrm{P}_{\langle\tau\rangle}\left(\mathbf{x}_{\mathrm{SNM}}(1)\right), \mathrm{P}_{\langle\tau\rangle}\left(\mathbf{x}_{\mathrm{SNM}}(2)\right), \ldots, \mathrm{P}_{\langle\tau\rangle}\left(\mathbf{x}_{\mathrm{SNM}}\left(N_{\mathrm{T}}\right)\right)\right]$.

Step 2: Calculate the upper bound on the SAP-wise mutual information: With $\mathrm{P}_{\langle\tau\rangle}\left(\mathbf{x}_{\mathrm{SNM}}(k)\right)$, we can refer to (17) and (21) to determine an upper bound on the SAP-wise mutual information as ${ }^{8}$

$$
\begin{aligned}
& \Xi_{\langle\tau\rangle}\left(\mathbf{x}_{\mathrm{SNM}}(k)\right) \\
& =\sum_{n \in \mathcal{N}_{\mathrm{S}}(k)} B \log _{2}\left(1+\frac{P_{\mathrm{T}}\left|h_{n}\right|^{2}}{k B N_{0}}\right)+D\left(\mathbf{P}_{\langle 1\rangle} \| \mathbf{P}_{\langle\tau\rangle}\right) \\
& \quad+\frac{1}{N_{\mathrm{T}}} \sum_{i=1}^{N_{\mathrm{T}}} \sum_{n=1}^{N_{\mathrm{S}}} D\left(f_{\mathrm{A} / \mathrm{I}}^{k}\left(y_{n}\right) \| f_{\mathrm{A} / \mathrm{I}}^{i}\left(y_{n}\right)\right),
\end{aligned}
$$

where

$$
D\left(\mathbf{P}_{\langle 1\rangle} \| \mathbf{P}_{\langle\tau\rangle}\right)=\frac{1}{N_{\mathrm{T}}} \sum_{i=1}^{N_{\mathrm{T}}} \log _{2}\left(\frac{1}{N_{\mathrm{T}} \mathrm{P}_{\langle\tau\rangle}\left(\mathbf{x}_{\mathrm{SNM}}(i)\right)}\right)
$$

\footnotetext{
${ }^{8}$ The derivation of this upper bound on the SAP-wise mutual information is presented in Appendix B for interested readers.
}

by the definition of Kullback-Leibler divergence for discrete probability distributions and (25);

$$
D\left(f_{\mathrm{A} / \mathrm{I}}^{k}\left(y_{n}\right) \| f_{\mathrm{A} / \mathrm{I}}^{i}\left(y_{n}\right)\right)=\log _{2}\left(\frac{\varrho_{n}^{i}}{\varrho_{n}^{k}}\right)-\left(1-\frac{\varrho_{n}^{k}}{\varrho_{n}^{i}}\right) \log _{2}(e)
$$

is derived between two complex Gaussian distributions in Appendix C.

Step 3: Calculate the upper bound on the weighted average of the SAP-wise mutual information: With the upper bound on the SAP-wise mutual information, we can determine an upper bound on the the weighted average of the SAP-wise mutual information by

$$
\begin{aligned}
\bar{C}_{\langle\tau\rangle}(\mathbf{H}) & =\underset{\mathbf{x}_{\mathrm{SNM}}(k)}{\mathbb{E}}\left\{\Xi_{\langle\tau\rangle}\left(\mathbf{x}_{\mathrm{SNM}}(k)\right)\right\} \\
& =\sum_{k=1}^{N_{\mathrm{T}}} \mathrm{P}_{\langle\tau\rangle}\left(\mathbf{x}_{\mathrm{SNM}}(k)\right) \Xi_{\langle\tau\rangle}\left(\mathbf{x}_{\mathrm{SNM}}(k)\right) .
\end{aligned}
$$

Step 4: Update the PUP vector: Different from the original PUP updating criterion relying on intermediate parameters proposed in [42], we directly utilize the upper bounds on the SAP-wise mutual information as references to update the PUP vector for the next iteration by

$$
\mathrm{P}_{\langle\tau+1\rangle}\left(\mathbf{x}_{\mathrm{SNM}}(k)\right)=\frac{2^{\Xi_{\langle\tau\rangle}\left(\mathbf{x}_{\mathrm{SNM}}(k)\right)}}{\sum_{i=1}^{N_{\mathrm{T}}} 2^{\Xi_{\langle\tau\rangle}\left(\mathbf{x}_{\mathrm{SNM}}(i)\right)}} .
$$

Step 5: Check the termination condition: With a preset termination threshold $\epsilon$, for $\tau \geq 2$, we check if the following termination condition is satisfied:

$$
\bar{C}_{\langle\tau\rangle}(\mathbf{H})-\bar{C}_{\langle\tau-1\rangle}(\mathbf{H})<\epsilon .
$$

If yes, the iterative process is terminated and the optimum is said to be converged. Then, we have the sub-optimal PUP vector

$$
\begin{aligned}
\mathbf{P}^{\&} & =\left[\mathrm{P}^{\&}\left(\mathbf{x}_{\mathrm{SNM}}(1)\right), \mathrm{P}^{\&}\left(\mathbf{x}_{\mathrm{SNM}}(2)\right), \ldots, \mathrm{P}^{\&}\left(\mathbf{x}_{\mathrm{SNM}}\left(N_{\mathrm{T}}\right)\right)\right]^{T} \\
& =\mathbf{P}_{\langle\tau\rangle} .
\end{aligned}
$$

Otherwise, the iterative process goes back to Step 2 and repeats until the above termination condition is satisfied.

\section{Closed-Form Approximations of the Optimum and the Channel Capacity}

\section{Setting of the Termination Threshold}

Here, we also propose an easy-to-implement approach to set the termination threshold $\epsilon$ so as to accurately approximate $\mathrm{P}^{*}$ by $\mathrm{P}^{\&}$ with a reasonable amount of computational resource. Recalling the basic properties of mutual information and entropy and assuming that all symbols carried on active subcarriers are Gaussian input with a total power constraint 
$P_{\mathrm{T}}$, we can derive a loose upper bound on channel capacity, denoted as $\Upsilon(\mathbf{H})$, as follows

$$
\begin{gathered}
C(\mathbf{H})<\Upsilon(\mathbf{H}) \doteq \underbrace{\max _{\mathbf{P}}\left\{-\sum_{k=1}^{N_{\mathrm{T}}} \mathrm{P}\left(\mathbf{x}_{\mathrm{SNM}}(k)\right) \log _{2}\left(\mathrm{P}\left(\mathbf{x}_{\mathrm{SNM}}(k)\right)\right)\right.}_{=\Upsilon_{\mathrm{SNM}}} \\
+\underbrace{\max _{\mathbf{P}}\left\{\sum_{k=1}^{N_{\mathrm{T}}} \mathrm{P}\left(\mathbf{x}_{\mathrm{SNM}}(k)\right) \sum_{n \in \mathcal{N}_{\mathrm{S}}(k)} B \log _{2}\left(1+\frac{P_{\mathrm{T}}\left|h_{n}\right|^{2}}{k B N_{0}}\right)\right\}}_{=\Upsilon_{\mathrm{APM}}(\mathbf{H})}
\end{gathered}
$$

By the method of Lagrange multipliers, we can easily find that the first term can be maximized once all SAPs are uniformly chosen, which yields

$$
\Upsilon_{\mathrm{SNM}}=\log _{2}\left(N_{\mathrm{T}}\right)
$$

Because of the linearity of $\Upsilon_{\mathrm{APM}}(\mathbf{H})$ with respect to probabilities, it is obvious that only a single SAP with the highest SAP-wise channel capacity resulted by the carried symbols on active subcarriers should be chosen to achieve the maximum of the second term. By this reasoning, we can derive

$$
\Upsilon_{\mathrm{APM}}(\mathbf{H})=\sum_{n \in \mathcal{N}_{\mathrm{S}}(\kappa)} B \log _{2}\left(1+\frac{P_{\mathrm{T}}\left|h_{n}\right|^{2}}{\kappa B N_{0}}\right),
$$

where

$$
\kappa=\underset{k}{\arg \max }\left\{\sum_{n \in \mathcal{N}_{\mathrm{S}}(k)} B \log _{2}\left(1+\frac{P_{\mathrm{T}}\left|h_{n}\right|^{2}}{k B N_{0}}\right)\right\} .
$$

Assuming a special case where all channels are faded in a completely correlative manner and with normalized channel power gains, i.e., $\left|h_{1}\right|^{2}=\left|h_{2}\right|^{2}=\cdots=\left|h_{N_{\mathrm{S}}}\right|^{2}=1$, we can explicitly derive the closed-form expression of $\Upsilon(\operatorname{diag}\{\mathbf{1}\})$ as $^{9}$

$$
\Upsilon(\operatorname{diag}\{\mathbf{1}\})=\log _{2}\left(N_{\mathrm{T}}\right)+N_{\mathrm{T}} B \log _{2}\left(1+\frac{P_{\mathrm{T}}}{N_{\mathrm{T}} B N_{0}}\right)
$$

To provide intuitive knowledge of this upper bound, we plot $\Upsilon(\operatorname{diag}\{\mathbf{1}\})$ with normalized bandwidth for different $N_{\mathrm{T}}$ and $P_{\mathrm{T}} / N_{0}$ in Fig. 4.

Obtaining such a loose upper bound $\Upsilon(\mathbf{H})$ on channel capacity, we can roughly estimate the order of magnitude for channel capacity. The order of magnitude for channel capacity can be used as prior knowledge and help to decide a proper value of the termination threshold $\epsilon$. Specifically, assuming the magnitude of $\Upsilon(\mathbf{H})$ to be $v$, with $\epsilon=10^{v-\varepsilon}$, we can achieve the accuracy of about $\varepsilon$ orders of magnitude lower than $\Upsilon$. As a rule of thumb, $\epsilon=10^{-5} \times \Upsilon(\mathbf{H})$ would maintain a balanced equilibrium between approximation accuracy and computational resource and can be used in simulations for most application scenarios [43].

\footnotetext{
${ }^{9}$ Interested readers might refer to Appendix D for the detailed derivation of $\Upsilon(\operatorname{diag}\{\mathbf{1}\})$.
}

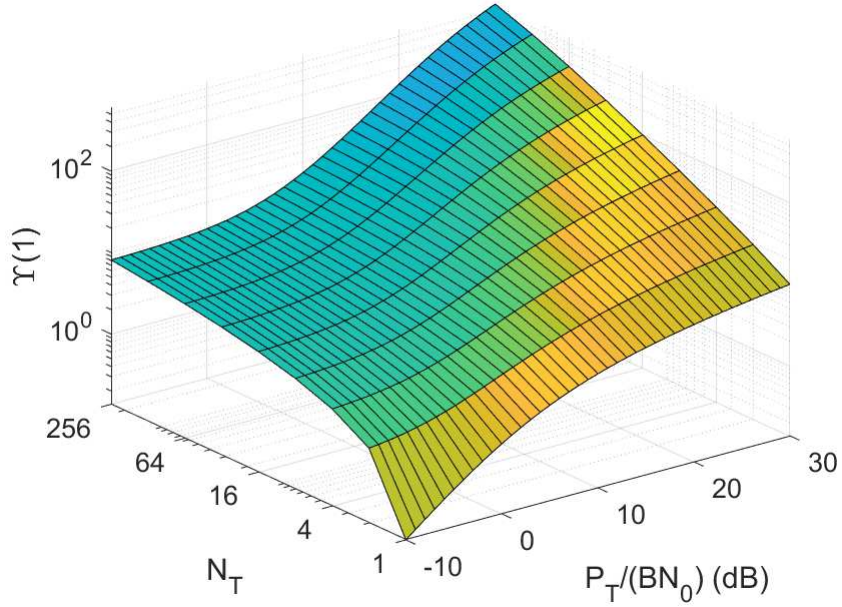

Fig. 4: $\Upsilon(\operatorname{diag}\{\mathbf{1}\})$ for different $N_{\mathrm{T}}$ and $P_{\mathrm{T}} / N_{0}$.

\section{E. Construction of Achievable PUP Vector}

Albeit with $\mathbf{P}^{\&}$ obtained by a sufficiently small $\epsilon$, we need to notice that such an optimized PUP vector might not be practically achievable when all incoming bits are assumed to be independently distributed and equiprobable in this paper. This is because the number of SAPs, i.e., $N_{\mathrm{T}}$, is a limited integer in OFDM-SNM systems, and each achievable PUP vector must be represented by a certain structure of a full binary tree comprised of $N_{\mathrm{T}}$ leaves [44]. This entropy coding constraint on the achievable PUP vector is imposed by the practical requirements of being uniquely and instantaneously decodable [33]. We hereby denote the full set of achievable PUP vectors by $\mathcal{U}\left(N_{\mathrm{T}}\right)$. As shown in [38], determining an appropriate achievable PUP vector $\mathbf{P}^{\#}$ from $\mathcal{U}\left(N_{\mathrm{T}}\right)$ through exhaustive search is nontrivial and with exponential computational complexity. The cardinality of $\mathcal{U}\left(N_{\mathrm{T}}\right)$ can be written as [38]

$$
\left|\mathcal{U}\left(N_{\mathrm{T}}\right)\right|=U\left(N_{\mathrm{T}}\right)=\sum_{t \in \mathcal{T}\left(N_{\mathrm{T}}-1\right)}\left(\begin{array}{c}
N_{\mathrm{T}} \\
\rho_{1}, \rho_{2}, \ldots, \rho_{N_{\mathrm{T}}-1}
\end{array}\right),
$$

where $\mathcal{T}\left(N_{\mathrm{T}}-1\right)$ is the reduced set of symmetric full binary trees with $N_{\mathrm{T}}-1$ internal nodes, i.e., $N_{\mathrm{T}}$ leaves associated with $N_{\mathrm{T}}$ legitimate SAPs; $\rho_{q}$ represents the number of leaves at level $q$ of tree $t$. The cardinality of $\mathcal{T}\left(N_{\mathrm{T}}-1\right)$ is the number of unique solution vectors $\left[\rho_{1}, \rho_{2}, \ldots, \rho_{N_{\mathrm{T}}-1}\right]^{T}$ satisfying the following equation set

$$
\left\{\begin{array}{l}
\sum_{q=1}^{N_{\mathrm{T}}-1} \rho_{q}(1 / 2)^{\rho_{q}}=1 \\
\sum_{q=1}^{N_{\mathrm{T}}-1} \rho_{q}=N_{\mathrm{T}} \\
0 \leq \rho_{q} \leq N_{\mathrm{T}}, \forall q=1,2, \ldots, N_{\mathrm{T}}-1 \\
\rho_{q} \in \mathbb{N}, \forall q=1,2, \ldots, N_{\mathrm{T}}-1
\end{array} .\right.
$$

As $\mathcal{U}\left(N_{\mathrm{T}}\right)$ is required for providing comparison benchmarks for numerical simulations, we adopt a simple but effective enumerating algorithm in Appendix $\mathrm{E}$ to generate $\mathcal{U}\left(N_{\mathrm{T}}\right)$ for small $N_{\mathrm{T}}$.

To facilitate the construction of achievable PUP vector, we employ Kullback-Leibler divergence as the distance measure 
between two probability distributions and define the optimal achievable PUP vector $\mathbf{P}^{\#}$ based on $\mathbf{P}^{\&}$ as

$$
\begin{aligned}
\mathbf{P}^{\#} & =\left[\mathrm{P}^{\#}\left(\mathbf{x}_{\mathrm{SNM}}(1)\right), \mathrm{P}^{\#}\left(\mathbf{x}_{\mathrm{SNM}}(2)\right), \ldots, \mathrm{P}^{\#}\left(\mathbf{x}_{\mathrm{SNM}}\left(N_{\mathrm{T}}\right)\right)\right]^{T} \\
& =\underset{\mathbf{P} \in \mathcal{U}\left(N_{\mathrm{T}}\right)}{\arg \min }\left\{D\left(\mathbf{P} \| \mathbf{P}^{\&}\right)\right\},
\end{aligned}
$$

where $D\left(\mathbf{P} \| \mathbf{P}^{\&}\right)$ can be explicitly written for our case as

$$
D\left(\mathbf{P} \| \mathbf{P}^{\&}\right)=\sum_{k=1}^{N_{\mathrm{T}}} \mathrm{P}\left(\mathbf{x}_{\mathrm{SNM}}(k)\right) \log _{2}\left(\frac{\mathrm{P}\left(\mathbf{x}_{\mathrm{SNM}}(k)\right)}{\mathrm{P}^{\&}\left(\mathbf{x}_{\mathrm{SNM}}(k)\right)}\right) \text {. }
$$

To obtain $\mathbf{P}^{\#}$ for OFDM-IM, the authors in [30] have utilized the principle of Huffman coding by sorting the SAPs by partial SAP-wise mutual information, which is equivalent to relying on the first term $\sum_{n \in \mathcal{N}_{\mathrm{S}}(k)} B \log _{2}\left(1+\frac{P_{\mathrm{T}}\left|h_{n}\right|^{2}}{k B N_{0}}\right)$ in (26). However, this is not optimal for both OFDM-IM and OFDM-SNM systems, because only the partial SAP-wise mutual information pertaining to the symbols carried on the active subcarriers is taken into account. The efficiency is even worse for OFDM-SNM, since the number of active subcarriers in OFDM-SNM systems is varying. Furthermore, in Huffman coding, ordering patterns by other associated references, including even the full SAP-wise mutual information, instead of their occurring probabilities has been proven to be less efficient [45], let alone the much higher computational complexity rendered by calculating mutual information for each pattern.

Hence, with the optimized PUP vector $\mathbf{P}^{\&}$, an efficient way to find out $\mathbf{P}^{\#}$ is to employ the Huffman coding associating $N_{\mathrm{T}}$ leaves labeled by their occurring probabilities $\left\{\mathrm{P}^{\&}\left(\mathbf{x}_{\mathrm{SNM}}(k)\right)\right\}_{k=1}^{N_{\mathrm{T}}}$ that represent $N_{\mathrm{T}}$ SAPs [38]. The detailed operational steps of the Huffman coding based achievable PUP vector construction procedure are presented as follows:

Step 1: Label SAPs: In accordance with Huffman coding, all SAPs are processed with labeled weight functions, which are the optimized PUPs $\left\{\mathrm{P}^{\&}\left(\mathbf{x}_{\mathrm{SNM}}(k)\right)\right\}_{k=1}^{N_{\mathrm{T}}}$ returned by the iterative algorithm detailed in Section IV-B.

Step 2: Order SAPs and their combinations: In the first round, all $N_{\mathrm{T}}$ SAPs should be ordered from the largest to the smallest in terms of $\mathrm{P}^{\&}\left(\mathrm{x}_{\mathrm{SNM}}(k)\right)$. Then, the two SAPs with the smallest and the next-to-smallest PUPs are merged to form an SAP combination labeled with the sum of the these two smallest probabilities. Subsequently, the SAP combination is treated as a whole in the next ordering round until the $\left(N_{\mathrm{T}}-\right.$ $1)$ th ordering round when all SAPs have been connected in a certain manner to form a full binary tree.

Step 3: Determine the achievable PUP vector: Examine the depth of the leaf associated with each connected SAP in the formed full binary tree, denoted as $d\left(\mathbf{x}_{\mathrm{SNM}}(k)\right)$, and determine the corresponding achievable PUP as

$$
\mathrm{P}^{\#}\left(\mathbf{x}_{\mathrm{SNM}}(k)\right)=2^{-d\left(\mathbf{x}_{\mathrm{SNM}}(k)\right)}, \forall k \in\left\{1,2, \ldots, N_{\mathrm{T}}\right\} .
$$

In contrast to the heuristic formalism of the binary tree aided projection in [38], we rigorously prove in Appendix F that the achievable PUP vector constructed in this way is the closest to $\mathbf{P}^{\&}$ in the sense of Kullback-Leibler divergence. The complete channel-dependent coding procedure, involving $\overline{\text { Algorithm } 1 \text { Complete channel-dependent coding procedure }}$ determining the achievable optimized PUP vector $\mathbf{P}^{\#}$.

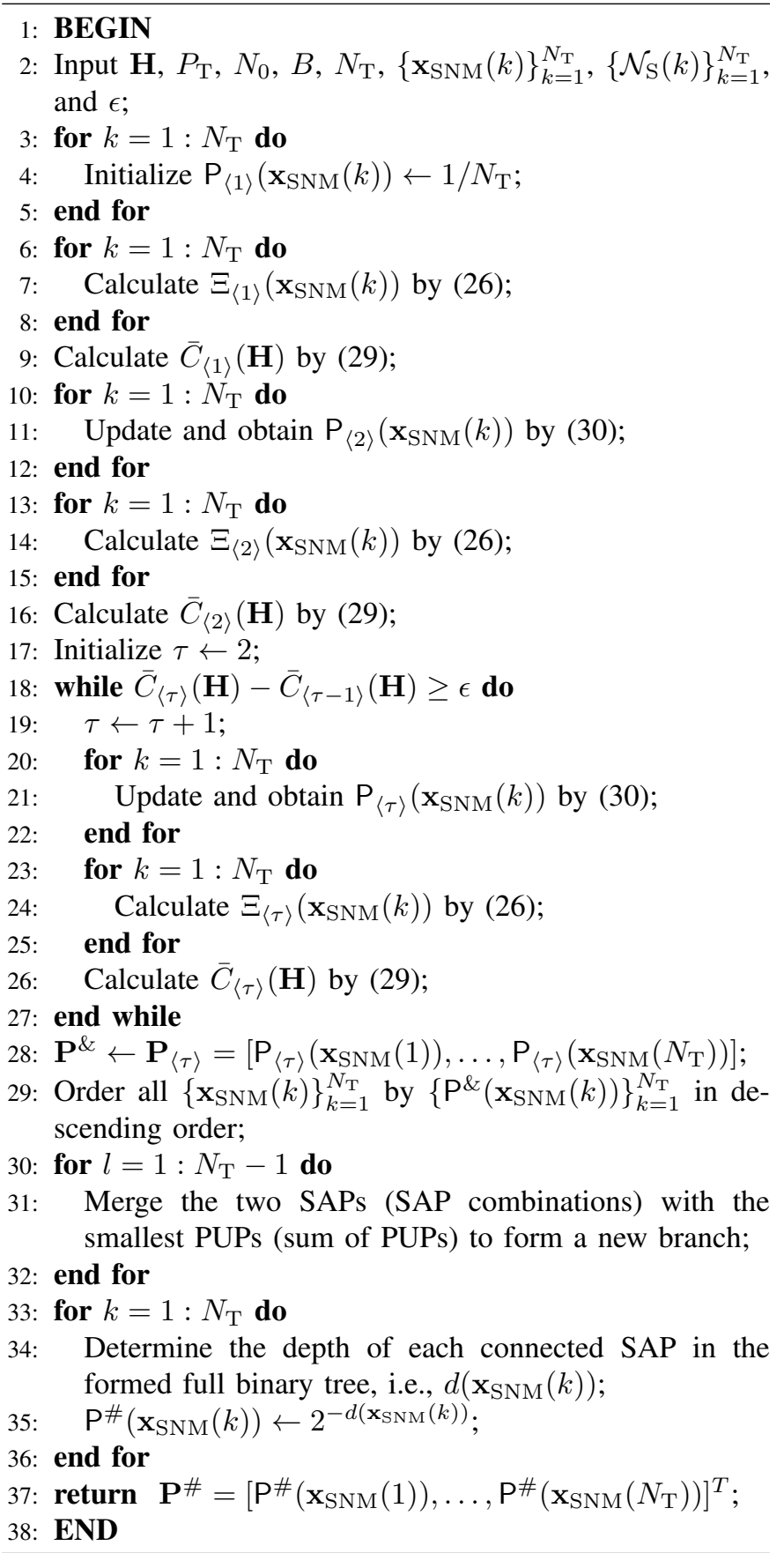

two stages of iterative optimization and Huffman coding, is specified in Algorithm 1 for clarity. We can pictorially illustrate the optimization procedure as well as the qualitative relation among $\mathbf{P}^{*}, \mathbf{P}^{\&}$, and $\mathbf{P}^{\#}$ in Fig. 5.

\section{NUMERICAL RESULTS AND DisCUSSION}

\section{A. Simulation Setups}

In the following simulations, we consider power and complexity constrained application scenarios and set up relatively simple OFDM-SNM systems with $N_{\mathrm{T}}=N_{\mathrm{S}}=2,4$. The 


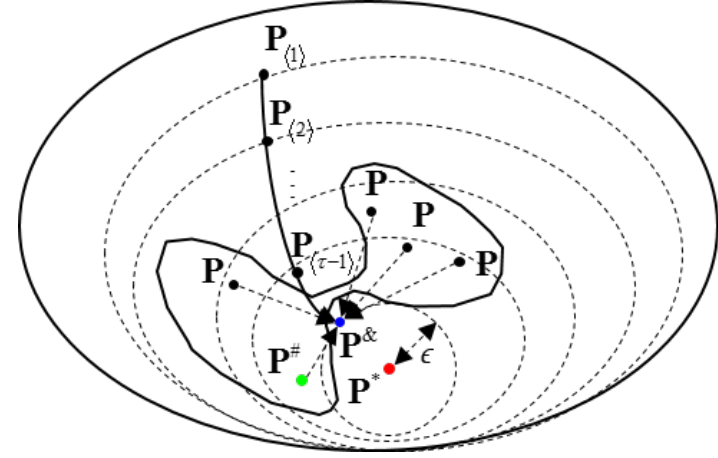

Fig. 5: Pictorial illustration of the entire optimization procedure with the qualitative relation among $\mathbf{P}^{*}, \mathbf{P}^{\&}$, and $\mathbf{P}^{\#}$.

bandwidth of each subcarrier $B$ and the noise PSD $N_{0}$ are normalized for simplicity. Without loss of generality, two channel fading models are taken into consideration for simulations:

- Normalized AWGN channel model: $\mathbf{H}=\operatorname{diag}\{\mathbf{1}\}$.

- Normalized Rayleigh fading channel model: $\mathbf{H}=$ $\operatorname{diag}\left\{h_{1}, h_{2}, \ldots, h_{N_{\mathrm{S}}}\right\}$, given $h_{n} \sim \mathcal{C N}(0,1), \forall n \in \mathcal{N}_{\mathrm{S}}$.

To capture the channel variations into consideration and focus on the average, we employ the ergodic channel capacity as a performance evaluation metric in this paper to inspect the effectiveness of the proposed methods and compare the simulation results. The ergodic channel capacity is given by

$$
C=\underset{\mathbf{H}}{\mathbb{E}}\{C(\mathbf{H})\},
$$

in which the $N_{\mathrm{S}}$-fold integral (c.f. (17)) is numerically calculated by Monte Carlo sampling averaging over $10^{2}$ samples [46]. The ergodic channel capacity illustrated in this section is approximated by averaging over $10^{4}$ repeated trials with independent channel realizations so as to reflect the statistical nature of OFDM-SNM and the statistical regularity by the law of large numbers.

For the normalized Rayleigh fading channel model, we also employ outage probability defined as

$$
P_{\text {out }}=\mathbb{P}\{C(\mathbf{H})<\varsigma\}
$$

to capture the probabilistic characteristics of OFDM-SNM applied in fast fading environment, where $\varsigma$ is a preset outage threshold signifying the reception capability and is normalized in the following simulations for simplicity.

Considering finite constellation inputs with a limited constellation order $M$, we employ the spectral efficiency to reflect and compare the information transmission capabilities among schemes with different settings. The spectral efficiency is defined as

$$
\begin{aligned}
R= & \underbrace{-\sum_{k=1}^{N_{\mathrm{T}}} \mathrm{P}\left(\mathbf{x}_{\mathrm{SNM}}(k)\right) \log _{2}\left(\mathrm{P}\left(\mathbf{x}_{\mathrm{SNM}}(k)\right)\right)}_{=R_{\mathrm{SNM}}} \\
& +\underbrace{\sum_{k=1}^{N_{\mathrm{T}}} \mathrm{P}\left(\mathbf{x}_{\mathrm{SNM}}(k)\right) k \log _{2}(M),}_{=R_{\mathrm{APM}}}
\end{aligned}
$$

in bit per channel use (bpcu), where $R_{\mathrm{SNM}}$ and $R_{\mathrm{APM}}$ represent the spectral efficiency brought by modulating input data over different SAPs and SCPs, respectively.

\section{B. Numerical Exploration of Ergodic Channel Capacity}

We carried out numerical simulations regarding ergodic channel capacity with respect to average signal-to-noise ratio over both AWGN channels and Rayleigh channels for two- and four-subcarrier systems. The numerical results corresponding to different system setups are shown in Figs. 6, 7, 8, and 9. By observing the presented numerical results, we can discover a series of important properties of OFDM-SNM and the proposed channel-dependent adaptive algorithms.

First of all, as all subchannels are statistically equivalent under the AWGN channel setup, thus, the channel-dependent SAP selection criterion of OFDM-SNM does not bring any performance advantage. Also, because of the reduced degree of freedom, there exists ergodic channel capacity underperformance of OFDM-SNM compared to classic OFDM. For this reason, the ergodic channel capacity yielded by classic OFDM serves as the upper bound over the entire signal-to-noise ratio region when the AWGN channel model is adopted. This is also in line with the observations and analysis regarding OFDM-IM given in [29].

Different from AWGN channels, the ergodic channel capacity yielded by OFDM-SNM can be higher than that of classic OFDM at low signal-to-noise ratio when the Rayleigh channel setup is utilized. This performance gain in the low power region is brought about by the channel-dependent SAP selection mechanism (c.f. (2)). These results align with the previous analysis that OFDM-SNM fits simple communication systems with transmit power constraints better.

More importantly, for both AWGN and Rayleigh channel setups, the ergodic channel capacities yielded by classic OFDM and the iterative algorithm converge at high signalto-noise ratio, which substantiates that the proposed iterative algorithm can approach the optimum. On the other hand, once we consider the practical achievability by processing the optimized PUP vector via the Huffman coding based scheme, an obvious theory-to-practice performance gap is yielded. For the case of $N_{\mathrm{T}}=2$, the processed PUP vector gives the same performance as by the uniform distribution, since there exists only one available structure for the full binary tree with two leaves. For the case of $N_{\mathrm{T}}=4$, the processed PUP vector is capable of outperforming the uniform distribution, while still exhibits an obvious loss compared to the optimized PUP vector directly yielded by the iterative algorithm.

In addition, further observing the PUP distributions for all cases clarifies how the ergodic channel capacity changes with different systems settings. Apparently, increasing transmit power will undermine the equilibrium among multiple PUPs. In the extreme case of infinite transmit power, the OFDM-SNM employing the optimized PUP vector given by the proposed iterative algorithm will become classic OFDM, since only a single SAP with all $N_{\mathrm{T}}$ available subcarriers activated will be chosen almost surely. This can be well explained by the phenomenon that with a sufficient amount of 

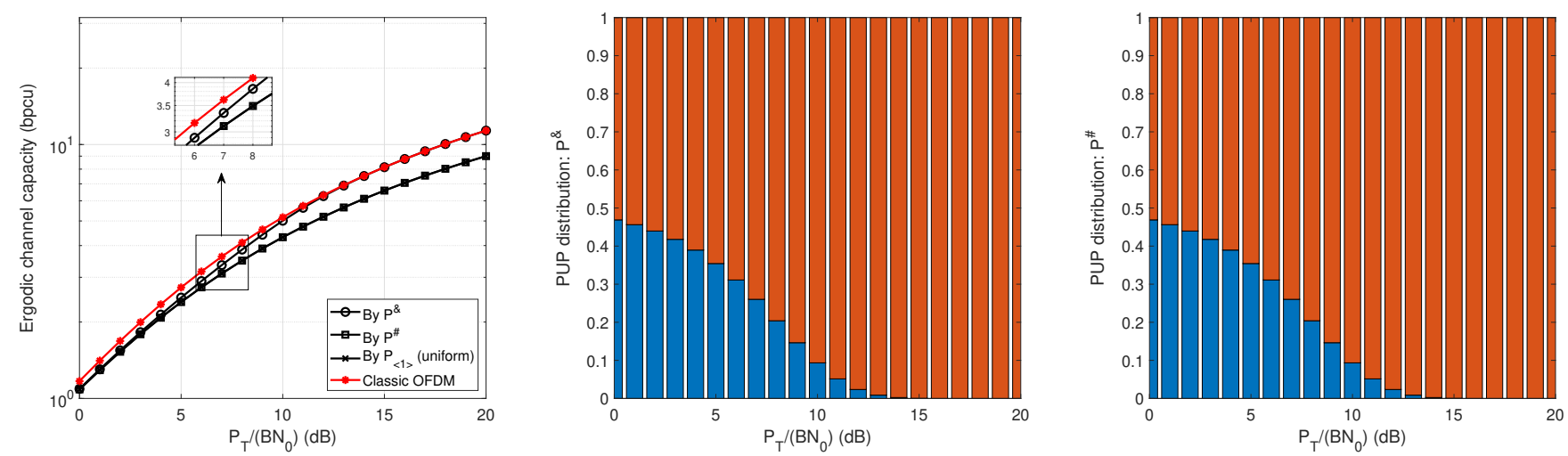

Fig. 6: Ergodic channel capacity and PUP distributions vs. average signal-to-noise ratio for AWGN channels, when $N_{\mathrm{T}}=2$.
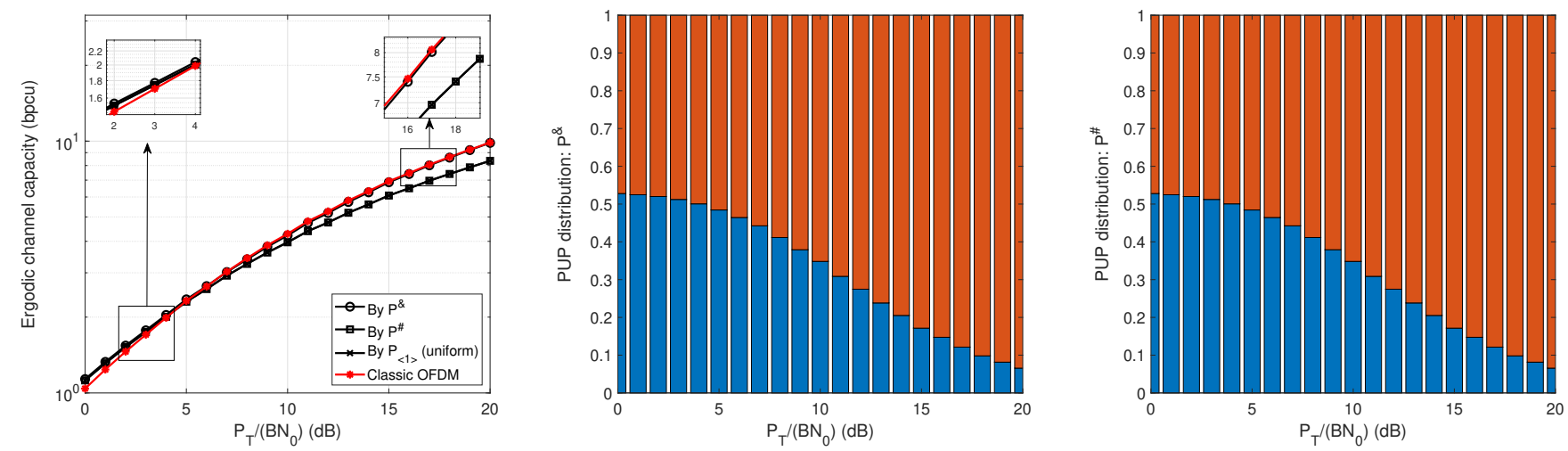

Fig. 7: Ergodic channel capacity and PUP distributions vs. average signal-to-noise ratio for Rayleigh channels, when $N_{\mathrm{T}}=2$.
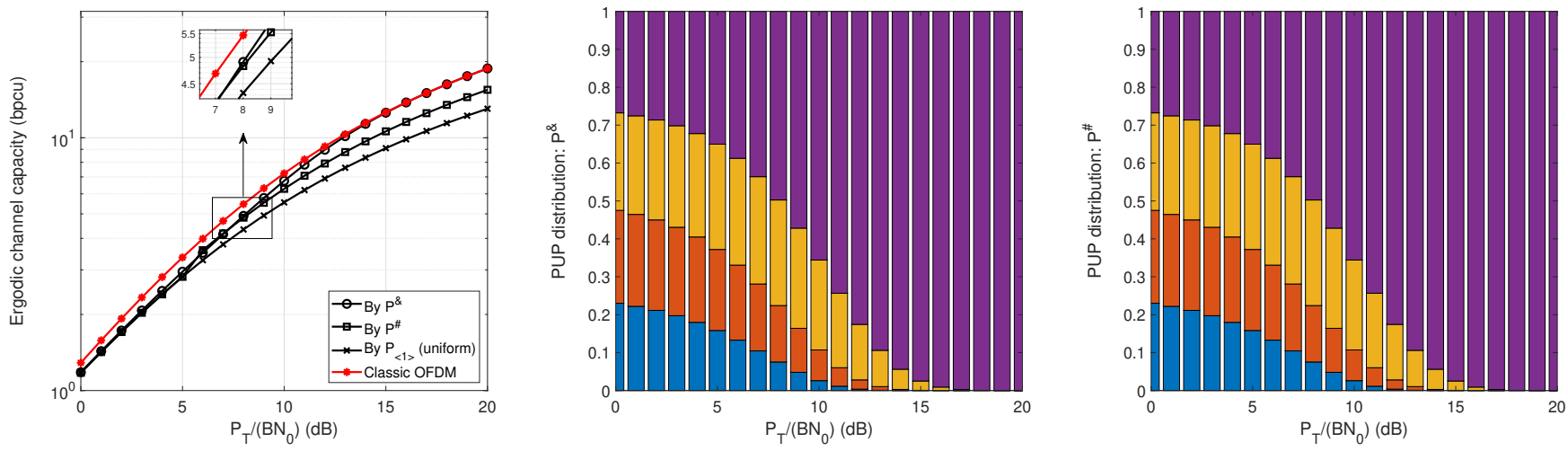

Fig. 8: Ergodic channel capacity and PUP distributions vs. average signal-to-noise ratio for AWGN channels, when $N_{\mathrm{T}}=4$.
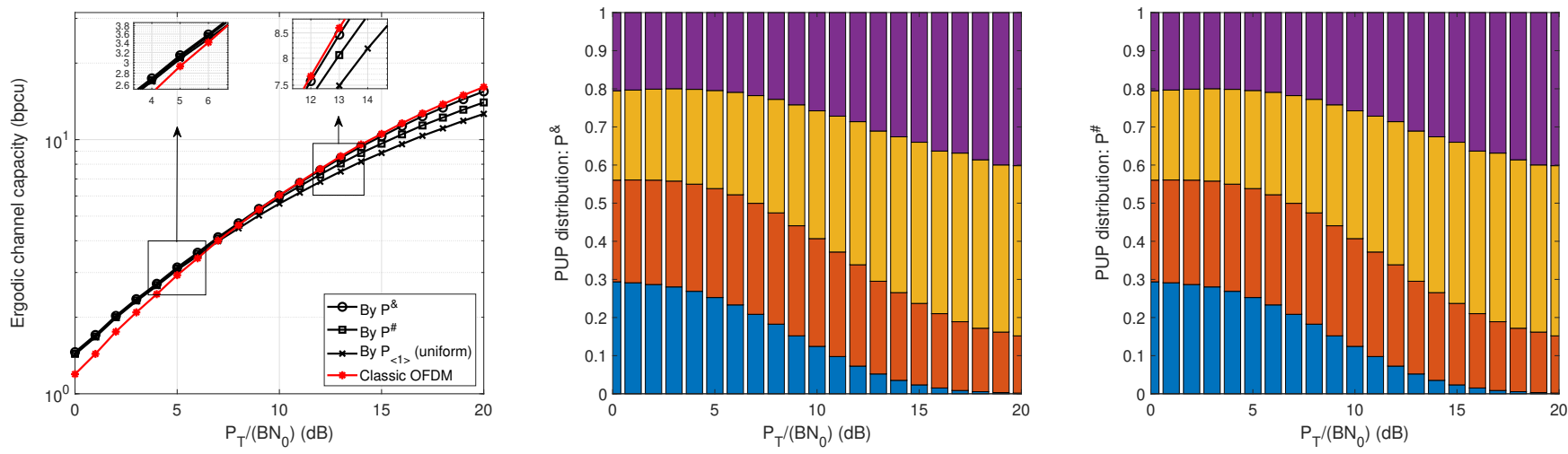

Fig. 9: Ergodic channel capacity and PUP distributions vs. average signal-to-noise ratio for Rayleigh channels, when $N_{\mathrm{T}}=4$. 
transmit power, all symbols carried on the subcarriers can be correctly received, which produces the capacity advantage over inactivating a subset of subcarriers but resorting to different SAPs for information transmission. Following this rationale, the effect of the transmit power in the low signal-to-noise ratio region can also be understood. As only a few symbols carried on subcarriers will be correctly received with less transmit power, the information conveyed by changed SAPs dominates, and thereby all PUPs turn to be uniformly chosen, adhering to the principle of maximum entropy. Because multi-path fading renders channel imbalance, which gives the room for improvement by the channel-dependent SAP selection criterion, the imbalance among different PUPs in the high signal-to-noise ratio can be mitigated when the Rayleigh channel setup is in use. In essence, transmit power serves as a weight between the information transmission potentials of SCPs and SAPs. As a result, with different transmit power, OFDM-SNM will resort to the proposed iterative algorithm and adapt the PUP distribution so as to achieve a higher ergodic channel capacity.

\section{Numerical Exploration of Outage Probability for Fast Fading Environment}

For the fast fading environment abiding the Rayleigh channel model, we simulated the outage performance under different system configurations and present the numerical results in Fig. 10. Note that because of the high consumption of computational power for simulating probability measures by Monte Carlo sampling, we set the simulations to collect $10^{2}$ outage events from an undetermined number of repeated trials with independent channel realizations for counting the numerical solutions of outage probability. As a result, the curves are not so smooth as those of ergodic channel capacity shown in the last subsection, albeit with adequate explanatory power.

In addition to the average measure, i.e., the ergodic channel capacity, the outage probability, as a probability measure, provides another view to inspect the proposed iterative algorithm and the Huffman coding based method. It is surprising that the outage probabilities yielded by OFDM-SNM with all three PUP distributions is lower than that of classic OFDM. The outage performance superiority is even more evident for a larger number of available subcarriers. These results imply that a higher degree of system reliability can be brought by OFDMSNM, even though the transmission capability measured by the ergodic channel capacity might not necessarily outperform. This sightly counterintuitive phenomenon can be interpreted by the fact that deep fading is better dealt with by avoiding activating unfavorable subcarriers in OFDM-SNM. This outcome is in line with the prior judgment that OFDM-SNM can better serve the IoT and MTC applications than classic OFDM, where the system reliability is taken much more seriously. Meanwhile, mapping the optimized PUP produced by the iterative algorithm to its achievable counterpart will only result in a trivial loss of outage performance, which is different from the case of ergodic channel capacity. Overall, from a perspective of system reliability, the effectiveness and efficiency of the proposed algorithms for OFDM-SNM have been corroborated.
Besides, the simulation results shown in Fig. 10 clearly reveal that no diversity gain can be harvested by OFDMSNM, albeit with the subcarrier assignment scheme relying on instantaneous CSI. Instead, a coding gain can be attained by exploiting channel knowledge and optimizing the sets of active subcarriers through (2). This is because that all subcarriers have been involved in the modulation process even though they are used with different priorities, but none of them has been eliminated from the modulation process by pre-selection. Such a unity-diversity-order property has been first reported and preliminary explained in [19]. With the information-theoretic analysis given in Section IV, we can have more in-depth understanding of the unity-diversity-order property of OFDMSNM.

\section{Numerical Exploration of Data Rate for Finite Constella- tion Input}

To reveal the effectiveness and efficiency of the proposed algorithms for practical communication systems using a finite constellation order $M$, we simulated the spectral efficiency for systems with $M=2$ (BPSK) and $M=4$ (QPSK) and illustrate the numerical results in Fig. 11. For comparison purposes, we derive the optimal PUP vector that maximizes the spectral efficiency by (45) in Appendix G, which is denoted as $\mathbf{P}^{@}$ in Fig. 11, serving as benchmarks.

From Fig. 11, it is clear that the curves associated with the optimized PUP vectors are able to hit the optimal benchmarks in the low-to-medium signal-to-noise power region when operating over AWGN channels. For both AWGN and Rayleigh channels, the spectral efficiency yielded by the optimized PUP vector is higher than that of plain OFDM, which verifies the effectiveness and efficiency of the proposed algorithms for practical communication systems with finite constellation orders. Moreover, it is worth noting that the spectral efficiency is not a monotone function of transmit power. This is because the transmit power alters the optimized PUP vector that maximizes the instantaneous channel capacity, while the optimized PUP vectors might not necessarily lead to the maximum spectral efficiency once the instantaneous channel capacity is maximized.

\section{E. Numerical Exploration of the Huffman Coding Based Achievable PUP Vector Construction Scheme}

To show the applicability of the Huffman coding based scheme, we further simulated the Kullback-Leibler divergences of three PUP distributions to the optimized one generated by the iterative algorithm:

- Huffman coding based scheme

- Enumerating method given in Appendix E

- Perfect binary tree construction corresponding to the uniform PUP distribution (the comparison benchmark)

The numerical results are given in Fig. 12 for both AWGN and Rayleigh channel setups with different numbers of subcarriers $N_{\mathrm{T}}$. In all cases, the pairs of curves pertaining to the Huffman coding based scheme and the enumerating method perfectly match each other, which verifies that the proposed Huffman coding based method is capable of generating the closest PUP distribution in the sense of Kullback-Leibler divergence. 

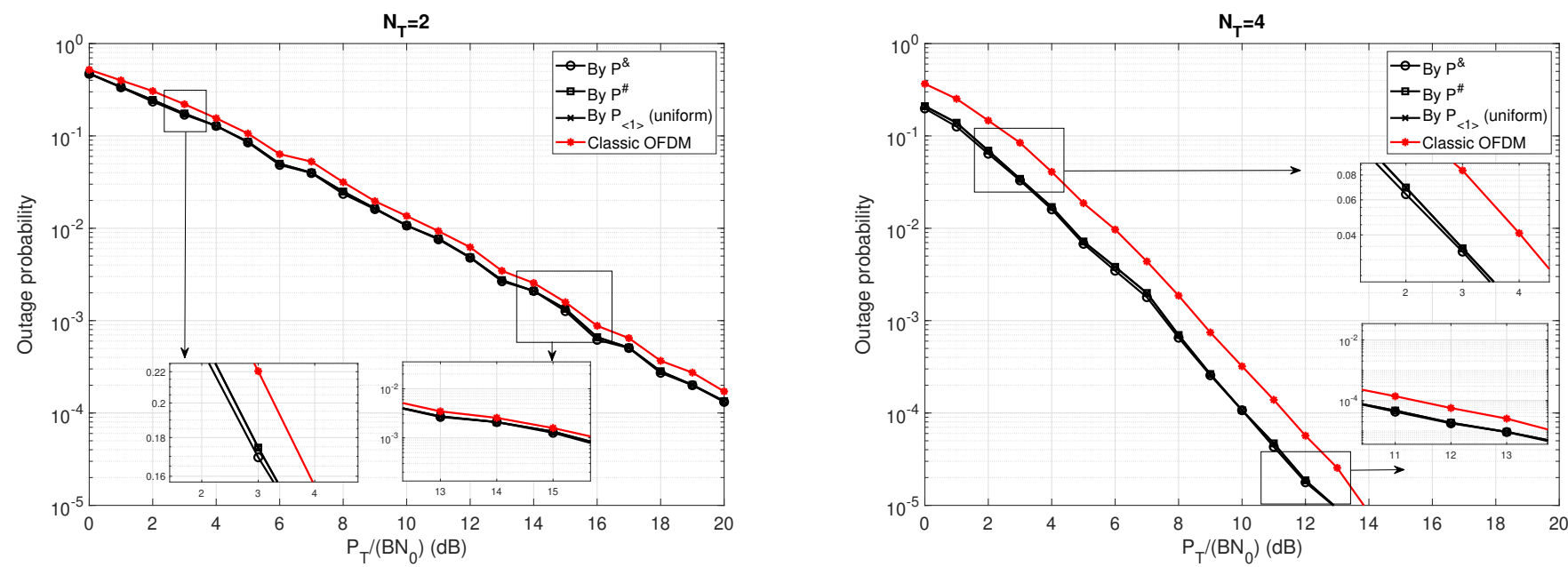

Fig. 10: Outage probability vs. average signal-to-noise ratio for Rayleigh channels for $N_{\mathrm{T}}=2,4$.
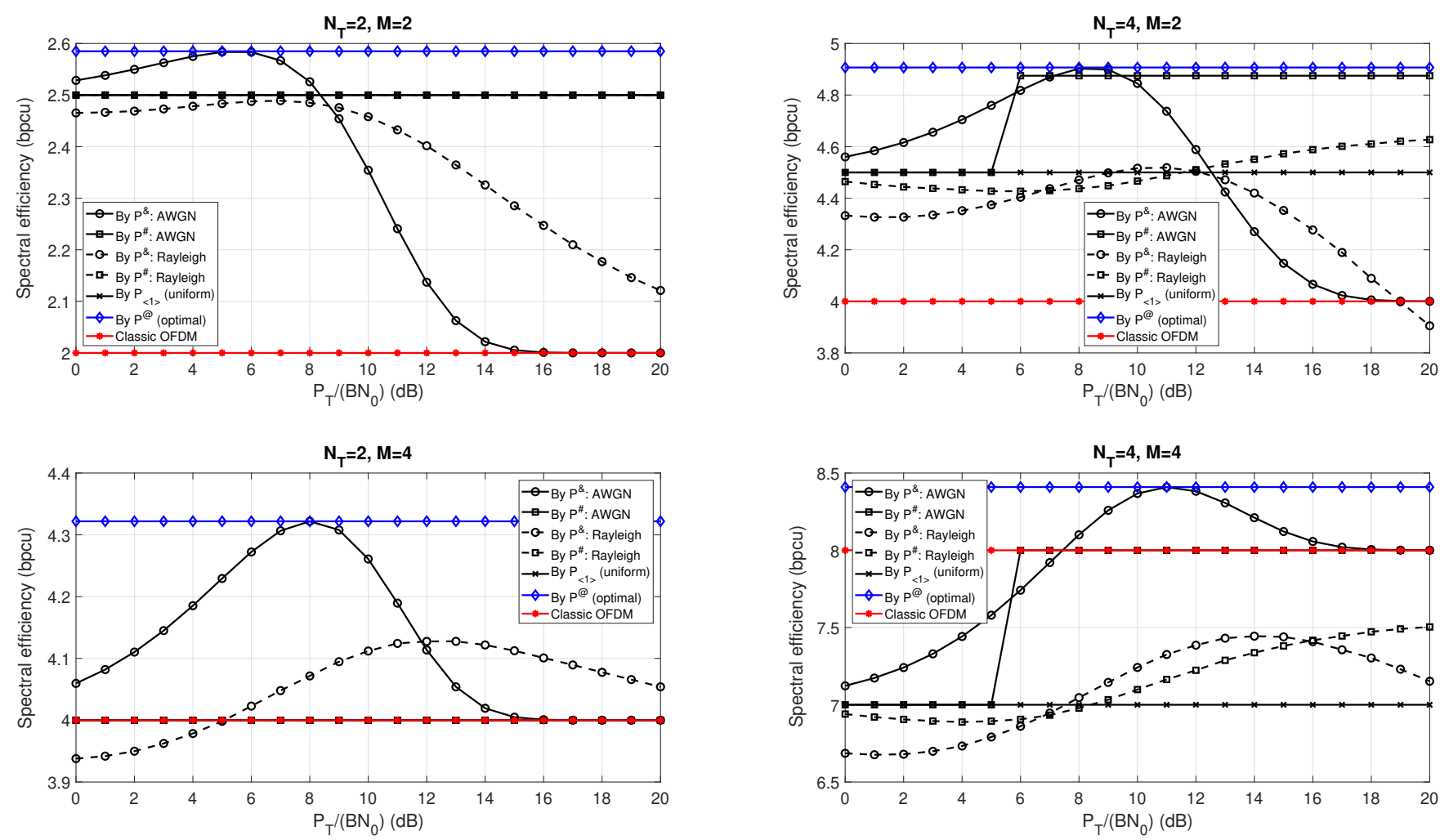

Fig. 11: Spectral efficiency vs. average signal-to-noise ratio for AWGN and Rayleigh channels with different $N_{\mathrm{T}}$ and $M$.

\section{CONCLUSion And Future Work}

In this paper, we studied the performance of OFDM-SNM from an information-theoretic perspective and confirmed its performance superiority over the plain OFDM scheme activating all subcarriers. Specifically, we derived an upper bound on the mutual information of OFDM-SNM in closed form by using the $\log$ sum inequality, based on which an easy-toimplement iterative algorithm has been developed to adapt the PUP vector for maximizing the instantaneous channel capacity. Then, we also investigated a series of practical issues of OFDM-SNM and ensure its practical relevance by proposing a Huffman coding based achievable PUP vector construction scheme. The analytical and numerical results exhibited in this paper verified the applicability of OFDM-SNM and performance superiority over plain OFDM for power-constrained and IoT/MTC applications. This paper sets a solid informationtheoretic cornerstone for the implementation of OFDM-SNM in next generation communication networks.

Having such a solid information-theoretic cornerstone, it is expected that more research attention will be devoted to OFDM-SNM in the near future. To promote this promising sparsity modulation paradigm, we summarize several worthwhile research directions that would be investigated as future work. First, as can be seen in Figs. 6-9, PUP distributions are 

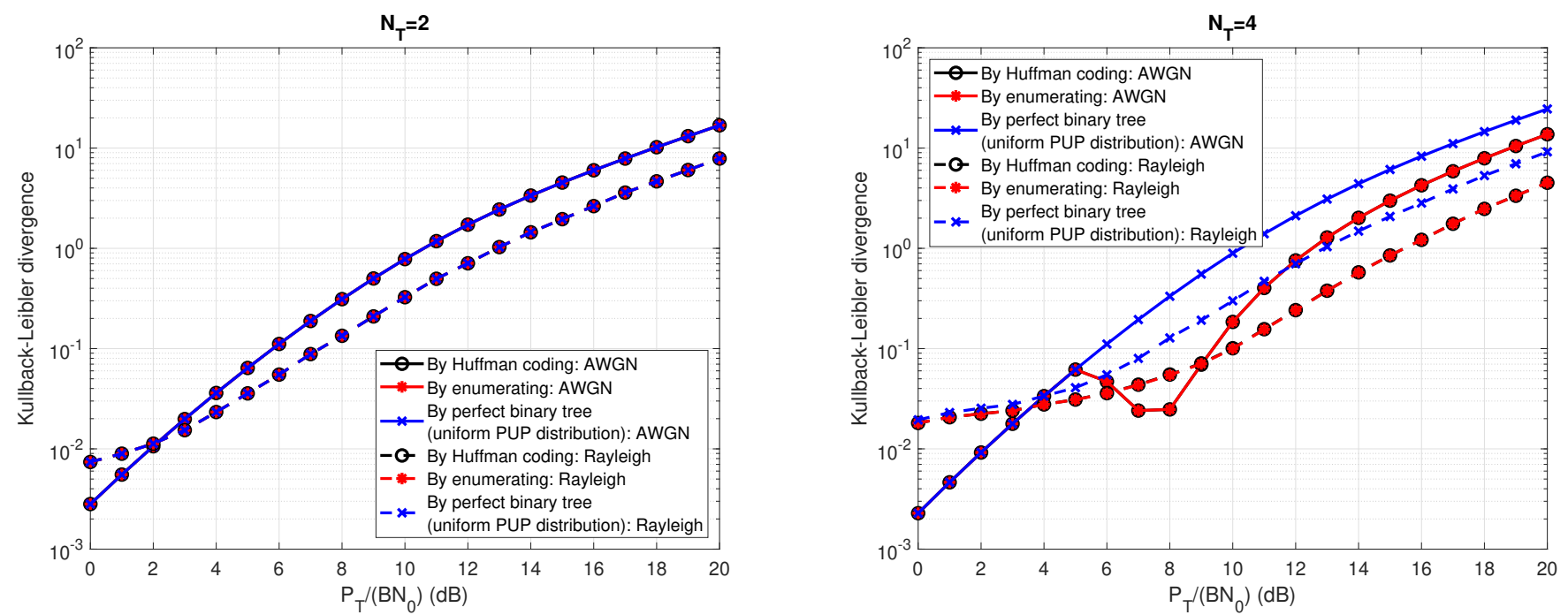

Fig. 12: Kullback-Leibler divergence vs. average signal-to-noise ratio for AWGN and Rayleigh channels for $N_{\mathrm{T}}=2,4$.

highly related to average signal-to-noise ratio and thereby the transmit power $P_{\mathrm{T}}$. Therefore, joint optimization considering both PUP and transmit power are of interest, which shall be analyzed as the foundation for the combination of OFDMSNM and OFDM with subcarrier power modulation (OFDMSPM) [47]. Meanwhile, OFDM-SNM can also be jointly applied with some advanced setups, e.g., dual-mode/multimode modulations; that is, the on/off states of subcarriers can be replaced by different constellation modes [13]; however, the in-depth performance analysis of such a combined scheme is still lacking and worth studying. Also, the detection of the entire OFDM-SNM block can be improved by extracting prior information from successive per-subcarrier detection, given a known subcarrier assignment scheme. The novel detector designs of OFDM-SNM would benefit from the latest advances in deep learning and distributed learning. The informationtheoretic analysis of multi-user application scenarios using OFDM-SNM is also worth investigating, and innovative mathematical tools might be developed to cope with the analytic challenges when multiple communication pairs are taken into account.

\section{APPENDIX A}

Proof of the Concavity of $I\left(\mathbf{x}_{\mathrm{SNM}}\left(K_{\mathrm{A}}\right) ; \mathbf{y}\left(K_{\mathrm{A}}\right)\right)$ IN $\mathbf{P}$

Substituting (23) into (24) yields

$$
\begin{aligned}
& I\left(\mathbf{x}_{\mathrm{SNM}}\left(K_{\mathrm{A}}\right) ; \mathbf{y}\left(K_{\mathrm{A}}\right)\right)=\sum_{k=1}^{N_{\mathrm{T}}} P\left(\mathbf{x}_{\mathrm{SNM}}\left(K_{\mathrm{A}}\right)\right) \\
& \times \int_{\mathbf{y}(k)} f_{\mathbf{y}}\left(\mathbf{y}_{k} \mid \mathbf{x}_{\mathrm{SNM}}\left(K_{\mathrm{A}}\right)=\mathbf{x}_{\mathrm{SNM}}(k)\right) \times \\
& \log _{2}\left(\frac{f_{\mathbf{y}}\left(\mathbf{y}_{k} \mid \mathbf{x}_{\mathrm{SNM}}\left(K_{\mathrm{A}}\right)=\mathbf{x}_{\mathrm{SNM}}(k)\right)}{\sum_{i=1}^{N_{\mathrm{T}}} P\left(\mathbf{x}_{\mathrm{SNM}}(i)\right) f_{\mathbf{y}}\left(\mathbf{y}(k) \mid \mathbf{x}_{\mathrm{SNM}}\left(K_{\mathrm{A}}\right)=\mathbf{x}_{\mathrm{SNM}}(i)\right)}\right) \mathrm{d} \mathbf{y}(k),
\end{aligned}
$$

which can be expended to two terms as

$$
\begin{aligned}
& I\left(\mathbf{x}_{\mathrm{SNM}}\left(K_{\mathrm{A}}\right) ; \mathbf{y}\left(K_{\mathrm{A}}\right)\right)=\sum_{k=1}^{N_{\mathrm{T}}} P\left(\mathbf{x}_{\mathrm{SNM}}\left(K_{\mathrm{A}}\right)\right) \\
& \times \int_{\mathbf{y}(k)} f_{\mathbf{y}}\left(\mathbf{y}_{k} \mid \mathbf{x}_{\mathrm{SNM}}\left(K_{\mathrm{A}}\right)=\mathbf{x}_{\mathrm{SNM}}(k)\right) \times \\
& \log _{2}\left(\frac{1}{\sum_{i=1}^{N_{\mathrm{T}}} P\left(\mathbf{x}_{\mathrm{SNM}}(i)\right) f_{\mathbf{y}}\left(\mathbf{y}(k) \mid \mathbf{x}_{\mathrm{SNM}}\left(K_{\mathrm{A}}\right)=\mathbf{x}_{\mathrm{SNM}}(i)\right)}\right) \mathrm{d} \mathbf{y}(k) \\
& +\sum_{k=1}^{N_{\mathrm{T}}} P\left(\mathbf{x}_{\mathrm{SNM}}\left(K_{\mathrm{A}}\right)\right) \int_{\mathbf{y}(k)} f_{\mathbf{y}}\left(\mathbf{y}_{k} \mid \mathbf{x}_{\mathrm{SNM}}\left(K_{\mathrm{A}}\right)=\mathbf{x}_{\mathrm{SNM}}(k)\right) \times \\
& \log _{2}\left(f_{\mathbf{y}}\left(\mathbf{y}_{k} \mid \mathbf{x}_{\mathrm{SNM}}\left(K_{\mathrm{A}}\right)=\mathbf{x}_{\mathrm{SNM}}(k)\right)\right) \mathrm{d} \mathbf{y}(k) .
\end{aligned}
$$

Given $f_{\mathbf{y}}\left(\mathbf{y}(k) \mid \mathbf{x}_{\mathrm{SNM}}\left(K_{\mathrm{A}}\right)=\mathbf{x}_{\mathrm{SNM}}(i)\right), i=1,2, \cdots, N_{\mathrm{T}}$, it is obvious that the first term of (47) is concave with respect to $\mathbf{P}$, just as the conventional form of the entropy function, and the second term is linear with respect to $\mathbf{P}$. Therefore, $I\left(\mathbf{x}_{\mathrm{SNM}}\left(K_{\mathrm{A}}\right) ; \mathbf{y}\left(K_{\mathrm{A}}\right)\right)$, as the sum of concave and linear terms, is also concave with respect to $\mathbf{P}$.

\section{APPENDIX B}

\section{DERIVATION OF THE UPPER BOUND ON THE SAP-WISE MUTUAL INFORMATION}

Assuming that Gaussian distributed symbols are conveyed on active subcarriers, we can have the SAP-wise mutual information for each $\operatorname{SAP} \mathbf{x}_{\mathrm{SNM}}(k)$ by (17) as

$$
\begin{aligned}
& \Lambda_{\langle\tau\rangle}\left(\mathbf{x}_{\mathrm{SNM}}(k)\right)=\sum_{n \in \mathcal{N}_{\mathrm{S}}(k)} B \log _{2}\left(1+\frac{P_{\mathrm{T}}\left|h_{n}\right|^{2}}{k B N_{0}}\right) \\
& +\int_{\mathbf{y}(k)} f_{\mathbf{y}}\left(\mathbf{y}(k) \mid \mathbf{x}_{\mathrm{SNM}}\left(K_{\mathrm{A}}\right)=\mathbf{x}_{\mathrm{SNM}}(k)\right) \\
& \times \underbrace{\log _{2}\left(\frac{f_{\mathbf{y}}\left(\mathbf{y}(k) \mid \mathbf{x}_{\mathrm{SNM}}\left(K_{\mathrm{A}}\right)=\mathbf{x}_{\mathrm{SNM}}(k)\right)}{f_{\mathbf{y}}(\mathbf{y}(k))} \mathrm{d} \mathbf{y}(k) .\right.}_{=\lambda_{\langle\tau\rangle}\left(\mathbf{x}_{\mathrm{SNM}}(k)\right)}
\end{aligned}
$$


However, to the best of our knowledge, the $N_{\mathrm{S}}$-fold integral in the second part of (48), denoted as $\lambda_{\langle\tau\rangle}\left(\mathbf{x}_{\mathrm{SNM}}(k)\right)$, cannot be solved analytically. The numerical evaluation of such a multiple integral is even tricky and time-consuming for conventional numerical platforms, e.g., MATLAB (up to triple integral can be numerically evaluated by the function integral3()). The incompetence of numerical evaluation of multiple integral hinders the optimization procedure. To enable the evaluation of $\lambda_{\langle\tau\rangle}\left(\mathbf{x}_{\mathrm{SNM}}(k)\right)$, we resort to the $\log$ sum inequality [39] and approximate $\lambda_{\langle\tau\rangle}\left(\mathbf{x}_{\mathrm{SNM}}(k)\right)$ by $\xi_{\langle\tau\rangle}\left(\mathbf{x}_{\mathrm{SNM}}(k)\right)$ in (49), which immediately leads to the upper bound on SAP-wise mutual information $\Xi_{\langle\tau\rangle}\left(\mathbf{x}_{\mathrm{SNM}}(k)\right)=$ $\sum_{n \in \mathcal{N}_{\mathrm{S}}(k)} B \log _{2}\left(1+\frac{P_{\mathrm{T}}\left|h_{n}\right|^{2}}{k B N_{0}}\right)+\xi_{\langle\tau\rangle}\left(\mathbf{x}_{\mathrm{SNM}}(k)\right) \geq$ $\Lambda_{\langle\tau\rangle}\left(\mathbf{x}_{\mathrm{SNM}}(k)\right)=\sum_{n \in \mathcal{N}_{\mathrm{S}}(k)} B \log _{2}\left(1+\frac{P_{\mathrm{T}}\left|h_{n}\right|^{2}}{k B N_{0}}\right)+$ $\lambda_{\langle\tau\rangle}\left(\mathbf{x}_{\mathrm{SNM}}(k)\right)$ as given in (26).

\section{APPENDIX C}

\section{DERIVATION OF THE KULlBACK-LEIBLER DiVERGENCE} BETWEEN TWO COMPLEX GAUSSIAN DisTRIBUTIONS

Proposition 1: Given two complex Gaussian distributions with zero mean denoted by $\mathcal{C N}\left(0, \varrho_{1}\right)$ and $\mathcal{C N}\left(0, \varrho_{2}\right)$, the Kullback-Leibler divergence between these two complex Gaussian distributions can be written as

$$
D\left(f_{1}(z) \| f_{2}(z)\right)=\log _{2}\left(\frac{\varrho_{2}}{\varrho_{1}}\right)-\left(1-\frac{\varrho_{1}}{\varrho_{2}}\right) \log _{2}(e),
$$

where $f_{1}(z)$ and $f_{2}(z)$ are the PDFs of complex Gaussian distributions $\mathcal{C N}\left(0, \varrho_{1}\right)$ and $\mathcal{C N}\left(0, \varrho_{2}\right)$.

Proof: According to the basic properties of the complex Gaussian distribution, we can explicitly express the PDF of a zero-mean complex Gaussian distribution as $f_{(\cdot)}(z)=$ $\frac{1}{\pi \varrho_{(\cdot)}} \exp \left(-\frac{|z|^{2}}{\varrho_{(\cdot)}}\right)$. By the definition of Kullback-Leibler divergence for continuous probability distributions, we can expand the Kullback-Leibler divergence between these two complex Gaussian distributions with zero mean as

$$
\begin{aligned}
& D\left(f_{1}(z) \| f_{2}(z)\right)=\int_{z \in \mathbb{C}} f_{1}(z) \log _{2}\left(\frac{f_{1}(z)}{f_{2}(z)}\right) \mathrm{d} z \\
& =\int_{z \in \mathbb{C}} f_{1}(z) \log _{2}\left(\frac{\varrho_{2}}{\varrho_{1}} \exp \left(-\left(\frac{1}{\varrho_{1}}-\frac{1}{\varrho_{2}}\right)|z|^{2}\right)\right) \mathrm{d} z \\
& =\log _{2}\left(\frac{\varrho_{2}}{\varrho_{1}}\right) \underbrace{\int_{z \in \mathbb{C}} f_{1}(z) \mathrm{d} z}_{=1}-\left(\frac{1}{\varrho_{1}}-\frac{1}{\varrho_{2}}\right) \log _{2}(e) \underbrace{\int_{z \in \mathbb{C}} f_{1}(z)|z|^{2} \mathrm{~d} z}_{=\varrho_{1}} \\
& =\log _{2}\left(\frac{\varrho_{2}}{\varrho_{1}}\right)-\left(1-\frac{\varrho_{1}}{\varrho_{2}}\right) \log _{2}(e) .
\end{aligned}
$$

Substituting $\varrho_{1}=\varrho_{n}^{k}$ and $\varrho_{2}=\varrho_{n}^{i}$ into (50) immediately results in the expression given in (28).

\section{APPENDIX D}

\section{DERIVATION OF $\Upsilon(\operatorname{diag}\{\mathbf{1}\})$}

Assuming $\left|h_{1}\right|^{2}=\left|h_{2}\right|^{2}=\cdots=\left|h_{N_{\mathrm{S}}}\right|^{2}=1$, we can reduce $\Upsilon_{\mathrm{APM}}(\operatorname{diag}\{\mathbf{1}\})$ by the symmetry of subcarriers to be

$$
\Upsilon_{\mathrm{APM}}(\operatorname{diag}\{\mathbf{1}\})=\Sigma[\kappa]=\kappa B \log _{2}\left(1+\frac{P_{\mathrm{T}}}{\kappa B N_{0}}\right) .
$$

We can then prove in the sequel that the number sequence $\Sigma[\kappa]$ is an increasing sequence with respect to $\kappa$, for $\kappa \geq 1$. First, we can release the domain of definition of $\kappa$ from positive integers to any positive real numbers. Then, we can regard $\Sigma[\kappa]$ as a function of $\kappa$ and have its first and second derivatives as

$$
\frac{\mathrm{d} \Sigma}{\mathrm{d} \kappa}=-\frac{B P_{\mathrm{T}} \log _{2}(e)}{\kappa B N_{0}+P_{\mathrm{T}}}+B \log _{2}\left(1+\frac{P_{\mathrm{T}}}{\kappa B N_{0}}\right),
$$

and

$$
\frac{\mathrm{d} \Sigma^{2}}{\mathrm{~d}^{2} \kappa}=-\frac{B P_{\mathrm{T}}^{2} \log _{2}(e)}{\kappa\left(\kappa B N_{0}+P_{\mathrm{T}}\right)^{2}} .
$$

Because $\frac{\mathrm{d} \Sigma^{2}}{\mathrm{~d}^{2} \kappa}<0$ for $k>0$, it can be known that $\frac{\mathrm{d} \Sigma}{\mathrm{d} \kappa}$ must be a monotone decreasing function of $\kappa$ and the minimum is approached for $\kappa \rightarrow \infty$. Consequently, we can obtain the relation infra

$$
\frac{\mathrm{d} \Sigma}{\mathrm{d} \kappa}>\lim _{\kappa \rightarrow \infty}\left\{\frac{\mathrm{d} \Sigma}{\mathrm{d} \kappa}\right\}=0,
$$

which indicates that $\Sigma[\kappa]$ is a monotone increasing function of $\kappa$ if $\kappa>0$, and so as an increasing number consequence when $\kappa$ takes positive integers. Therefore, When $\kappa=N_{\mathrm{T}}$, the maximum of the SAP-wise channel capacity resulted by the carried symbols on active subcarriers is achieved, and (37) is thereby proven.

\section{APPENDIX E \\ EnUmerating Algorithm Generating $\mathcal{U}\left(N_{\mathrm{T}}\right)$ FOR SMALL $N_{\mathrm{T}}$}

We present the enumerating algorithm generating $\mathcal{U}\left(N_{\mathrm{T}}\right)$ with all admissible probability distributions by deletion. As the enumerating algorithm by deletion is in fact an exhaustive search, which will result in exponentially increasing computational complexity with $N_{\mathrm{T}}$, this algorithm is only suited for small $N_{\mathrm{T}}$ and used in this paper to provide benchmarks for comparison purposes only.

To generate $\mathcal{U}\left(N_{\mathrm{T}}\right)$, we first need to have $\mathcal{T}\left(N_{\mathrm{T}}-1\right)$ and then permute all $N_{\mathrm{T}}$ SAPs with different depths of the leaves for all trees in $\mathcal{T}\left(N_{\mathrm{T}}-1\right)$. According to (39), a tree structure can be characterized by a unique solution vector $\left[\rho_{1}, \rho_{2}, \ldots, \rho_{N_{\mathrm{T}}-1}\right]^{T}$. Based on this reasoning, the generating algorithm of $\mathcal{T}\left(N_{\mathrm{T}}-1\right)$ can be designed as shown in Algorithm 2, where permn $\left(\mathbf{V}, N_{\mathrm{T}}-1\right)$ returns all permutations with repetition of $N_{\mathrm{T}}-1$ entries taken from $\mathbf{V}$.

Having obtained $\mathcal{T}\left(N_{\mathrm{T}}-1\right)$, we can now construct the set of all admissible probability distributions $\mathcal{U}\left(N_{\mathrm{T}}\right)$ by permuting distinguishable groups of leaves with different depths for all trees in $\mathcal{T}\left(N_{\mathrm{T}}-1\right)$. The detailed procedures for generating $\mathcal{U}\left(N_{\mathrm{T}}\right)$ from $\mathcal{T}\left(N_{\mathrm{T}}-1\right)$ by deletion are presented in Algorithm 3 , where $\operatorname{catc}(\cdot, \cdot)$ and $\operatorname{catr}(\cdot, \cdot)$ return the concatenated matrix/vector of the two inputs by column and row, respectively; perms $(\cdot)$ returns a matrix containing all permutations of the elements of the input vector without repetition; unique $(\cdot)$ deletes the repeated rows of the input matrix and thereby ensures that all rows are unique. 


$$
\begin{aligned}
& \lambda_{\langle\tau\rangle}\left(\mathbf{x}_{\mathrm{SNM}}(k)\right)=\int_{\mathbf{y}(k)} \sum_{i=1}^{N_{\mathrm{T}}}\left(\frac{1}{N_{\mathrm{T}}} f_{\mathbf{y}}\left(\mathbf{y}(k) \mid \mathbf{x}_{\mathrm{SNM}}\left(K_{\mathrm{A}}\right)=\mathbf{x}_{\mathrm{SNM}}(k)\right)\right) \\
& \times \log _{2}\left(\frac{\sum_{i=1}^{N_{\mathrm{T}}}\left(\frac{1}{N_{\mathrm{T}}} f_{\mathbf{y}}\left(\mathbf{y}(k) \mid \mathbf{x}_{\mathrm{SNM}}\left(K_{\mathrm{A}}\right)=\mathbf{x}_{\mathrm{SNM}}(k)\right)\right)}{\sum_{i=1}^{N_{\mathrm{T}}} \mathrm{P}_{\langle\tau\rangle}\left(\mathbf{x}_{\mathrm{SNM}}(i)\right) f_{\mathbf{y}}\left(\mathbf{y}(k) \mid \mathbf{x}_{\mathrm{SNM}}\left(K_{\mathrm{A}}\right)=\mathbf{x}_{\mathrm{SNM}}(i)\right)}\right) \mathrm{d} \mathbf{y}(k) \\
& \leq \xi_{\langle\tau\rangle}\left(\mathbf{x}_{\mathrm{SNM}}(k)\right)=\int_{\mathbf{y}(k)} \sum_{i=1}^{N_{\mathrm{T}}} \frac{1}{N_{\mathrm{T}}} f_{\mathbf{y}}\left(\mathbf{y}(k) \mid \mathbf{x}_{\mathrm{SNM}}\left(K_{\mathrm{A}}\right)=\mathbf{x}_{\mathrm{SNM}}(k)\right) \\
& \times \log _{2}\left(\frac{\frac{1}{N_{\mathrm{T}}} f_{\mathbf{y}}\left(\mathbf{y}(k) \mid \mathbf{x}_{\mathrm{SNM}}\left(K_{\mathrm{A}}\right)=\mathbf{x}_{\mathrm{SNM}}(k)\right)}{\mathrm{P}_{\langle\tau\rangle}\left(\mathbf{x}_{\mathrm{SNM}}(i)\right) f_{\mathbf{y}}\left(\mathbf{y}(k) \mid \mathbf{x}_{\mathrm{SNM}}\left(K_{\mathrm{A}}\right)=\mathbf{x}_{\mathrm{SNM}}(i)\right)}\right) \mathrm{d} \mathbf{y}(k) \\
& =\int_{\mathbf{y}(k)} \sum_{i=1}^{N_{\mathrm{T}}} \frac{1}{N_{\mathrm{T}}}\left(\prod_{n \in \mathcal{N}_{\mathrm{S}}(k)} f_{\mathrm{A}}^{k}\left(y_{n}\right)\right)\left(\prod_{n \notin \mathcal{N}_{\mathrm{S}}(k)} f_{\mathrm{I}}^{k}\left(y_{n}\right)\right) \\
& \times \log _{2}\left(\frac{\left(\prod_{n \in \mathcal{N}_{\mathrm{S}}(k)} f_{\mathrm{A}}^{k}\left(y_{n}\right)\right)\left(\prod_{n \notin \mathcal{N} \mathrm{S}(k)} f_{\mathrm{I}}^{k}\left(y_{n}\right)\right)}{N_{\mathrm{T}} \mathrm{P}_{\langle\tau\rangle}\left(\mathbf{x}_{\mathrm{SNM}}(i)\right)\left(\prod_{n \in \mathcal{N}_{\mathrm{S}}(i)} f_{\mathrm{A}}^{i}\left(y_{n}\right)\right)\left(\prod_{n \notin \mathcal{N}_{\mathrm{S}}(i)} f_{\mathrm{I}}^{i}\left(y_{n}\right)\right)}\right) \mathrm{d} \mathbf{y}(k) \\
& =D\left(\mathbf{P}_{\langle 1\rangle} \| \mathbf{P}_{\langle\tau\rangle}\right)+\frac{1}{N_{\mathrm{T}}} \sum_{i=1}^{N_{\mathrm{T}}} \sum_{n=1}^{N_{\mathrm{S}}} D\left(f_{\mathrm{A} / \mathrm{I}}^{k}\left(y_{n}\right) \| f_{\mathrm{A} / \mathrm{I}}^{i}\left(y_{n}\right)\right)
\end{aligned}
$$

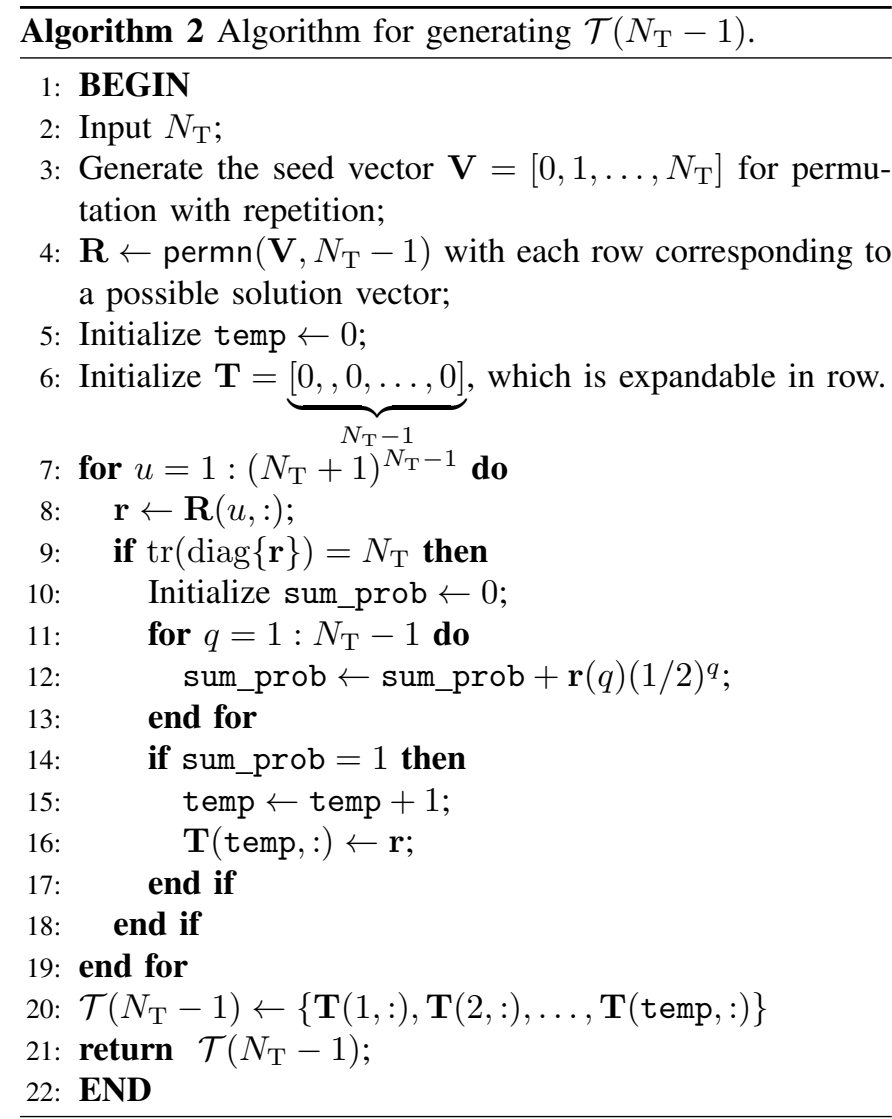

\section{APPENDIX F}

Proof of the Closest Nature in the Sense of KULLBACK-LEIBLER DIVERGENCE

By the definition of Kullback-Leibler divergence, we can expend $D\left(\mathbf{P}^{\&} \| \mathbf{P}^{\#}\right)$ as

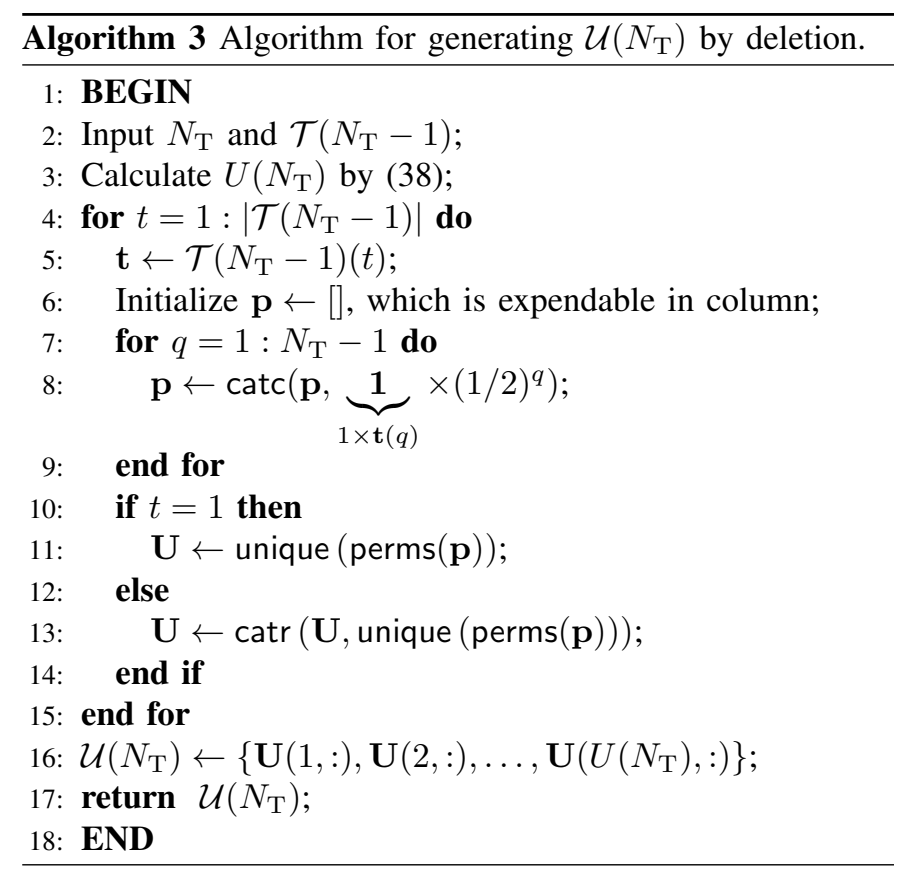

$$
\begin{aligned}
D\left(\mathbf{P}^{\&} \| \mathbf{P}^{\#}\right)= & \sum_{k=1}^{N_{\mathrm{T}}} \mathrm{P}^{\&}\left(\mathbf{x}_{\mathrm{SNM}}(k)\right) \log _{2}\left(\frac{\mathrm{P}^{\&}\left(\mathbf{x}_{\mathrm{SNM}}(k)\right)}{\mathrm{P}^{\#}\left(\mathbf{x}_{\mathrm{SNM}}(k)\right)}\right) \\
= & \sum_{k=1}^{N_{\mathrm{T}}} \mathrm{P}^{\&}\left(\mathbf{x}_{\mathrm{SNM}}(k)\right) \log _{2} \mathrm{P}^{\&}\left(\mathbf{x}_{\mathrm{SNM}}(k)\right) \\
& -\sum_{k=1}^{N_{\mathrm{T}}} \mathrm{P}^{\&}\left(\mathbf{x}_{\mathrm{SNM}}(k)\right) \log _{2} \mathrm{P}^{\#}\left(\mathbf{x}_{\mathrm{SNM}}(k)\right) .
\end{aligned}
$$


Substituting $\mathrm{P}^{\#}\left(\mathbf{x}_{\mathrm{SNM}}(k)\right)=2^{-d\left(\mathbf{x}_{\mathrm{SNM}}(k)\right)}$ into (56) gives

$$
D\left(\mathbf{P}^{\&} \| \mathbf{P}^{\#}\right)=\underbrace{\sum_{k=1}^{N_{\mathrm{T}}} \mathbf{P}^{\&}\left(\mathbf{x}_{\mathrm{SNM}}(k)\right) d\left(\mathbf{x}_{\mathrm{SNM}}(k)\right)}_{=\bar{L}}-H\left(\mathbf{P}^{\&}\right),
$$

where $\bar{L}$ denotes the expected code length. As proven by Cover and Thomas in [39], Hamming codes are the optimal instantaneous codes with the minimum expected length. Therefore, it is proven that $\mathbf{P}^{\#}$ is the closest to $\mathbf{P}^{\&}$ in the sense of Kullback-Leibler divergence.

\section{APPENDIX G \\ DERIVATION OF THE OPTIMAL PUP VECTOR MAXIMIZING THE SPECTRAL EFFICIENCY}

According (45), we can construct the following Lagrange function by the concavity of spectral information with respect to PUPs:

$$
\begin{aligned}
\mathcal{L}(\mathbf{P}, \omega)= & \sum_{k=1}^{N_{\mathrm{T}}} \mathrm{P}\left(\mathbf{x}_{\mathrm{SNM}}(k)\right)\left(-\log _{2}\left(\mathrm{P}\left(\mathbf{x}_{\mathrm{SNM}}(k)\right)\right)+k \log _{2}(M)\right) \\
& -\omega\left(1-\sum_{i=1}^{N_{\mathrm{T}}} \mathrm{P}\left(\mathbf{x}_{\mathrm{SNM}}(i)\right)\right),
\end{aligned}
$$

where $\omega$ is the Lagrange multiplier. Taking the partial derivative of $\mathcal{L}(\mathbf{P}, \omega)$ with respect to $\left\{\mathrm{P}\left(\mathrm{x}_{\mathrm{SNM}}(k)\right)\right\}$ and $\omega$ gives

$$
\begin{aligned}
\frac{\partial \mathcal{L}(\mathbf{P}, \omega)}{\partial \mathrm{P}\left(\mathbf{x}_{\mathrm{SNM}}(k)\right)}= & -\log _{2}\left(\mathrm{P}\left(\mathbf{x}_{\mathrm{SNM}}(k)\right)\right)+k \log _{2}(M) \\
& -\log _{2}(e)+w
\end{aligned}
$$

and

$$
\frac{\partial \mathcal{L}(\mathbf{P}, \omega)}{\partial \omega}=-1+\sum_{i=1}^{N_{\mathrm{T}}} \mathrm{P}\left(\mathbf{x}_{\mathrm{SNM}}(i)\right)
$$

Let $\frac{\partial \mathcal{L}(\mathbf{P}, \omega)}{\partial \mathrm{P}\left(\mathbf{x}_{\mathrm{SNM}}(k)\right)}=0$, and we can obtain

$$
\mathrm{P}^{@}\left(\mathbf{x}_{\mathrm{SNM}}(k)\right)=\frac{2^{\omega} M^{k}}{e},
$$

which can be substitute into $\frac{\partial \mathcal{L}(\mathbf{P}, \omega)}{\partial \omega}=0$ to yield

$$
\omega=\log _{2}\left(e\left(\sum_{i=1}^{N_{\mathrm{T}}} M^{i}\right)^{-1}\right) .
$$

Finally, substituting (62) back to (61) produces the optimal PUP maximizing the spectral efficiency of OFDM-SNM as

$$
\mathrm{P}^{@}\left(\mathbf{x}_{\mathrm{SNM}}(k)\right)=M^{k}\left(\sum_{i=1}^{N_{\mathrm{T}}} M^{i}\right)^{-1} .
$$

\section{ACKNOWLEDGMENT}

We thank the anonymous reviewers for their constructive comments, which helped us improve the quality of the paper.

\section{REFERENCES}

[1] K. David and H. Berndt, "6G vision and requirements: Is there any need for beyond 5G?" IEEE Vehicular Technology Magazine, vol. 13, no. 3, pp. 72-80, Sept. 2018.

[2] M. Mohamed, A. Samarah, and M. F. Allah, "Implementation of the OFDM physical layer using FPGA," International Journal of Computer Science Issues, vol. 9, no. 2, pp. 612-618, Mar. 2012.

[3] J. Vlaovic, S. Rimac-Drlje, and G. Horvat, "Overview of OFDM channel estimation techniques for DVB-T2 systems," in Proc. IEEE SST, Osijek, Croatia, Oct. 2016, pp. 75-80.

[4] J. G. Andrews, S. Buzzi, W. Choi, S. V. Hanly, A. Lozano, A. C. K. Soong, and J. C. Zhang, "What will 5G be?" IEEE Journal on Selected Areas in Communications, vol. 32, no. 6, pp. 1065-1082, June 2014.

[5] S. Dang, O. Amin, B. Shihada, and M.-S. Alouini, "What should 6G be?" Nature Electronics, vol. 3, no. 1, pp. 20-29, Jan. 2020.

[6] E. Basar, U. Aygolu, E. Panayırcı, and H. V. Poor, "Orthogonal frequency division multiplexing with index modulation," IEEE Transactions on Signal Processing, vol. 61, no. 22, pp. 5536-5549, Nov. 2013.

[7] E. Basar, M. Wen, R. Mesleh, M. Di Renzo, Y. Xiao, and H. Haas, "Index modulation techniques for next-generation wireless networks," IEEE Access, vol. 5, pp. 16693-16746, 2017.

[8] X. Cheng, M. Zhang, M. Wen, and L. Yang, "Index modulation for 5G: Striving to do more with less," IEEE Wireless Communications, vol. 25, no. 2, pp. 126-132, Apr. 2018.

[9] J. M. Hamamreh, E. Basar, and H. Arslan, "OFDM-subcarrier index selection for enhancing security and reliability of 5G URLLC services," IEEE Access, vol. 5, pp. 25 863-25875, 2017.

[10] A. M. Jaradat, J. M. Hamamreh, and H. Arslan, "Modulation options for OFDM-based waveforms: Classification, comparison, and future directions," IEEE Access, vol. 7, pp. 17263-17278, 2019.

[11] R. Fan, Y. J. Yu, and Y. L. Guan, "Generalization of orthogonal frequency division multiplexing with index modulation," IEEE Transactions on Wireless Communications, vol. 14, no. 10, pp. 5350-5359, Oct. 2015

[12] M. Wen, B. Ye, E. Basar, Q. Li, and F. Ji, "Enhanced orthogonal frequency division multiplexing with index modulation," IEEE Transactions on Wireless Communications, vol. 16, no. 7, pp. 4786-4801, July 2017

[13] M. Wen, E. Basar, Q. Li, B. Zheng, and M. Zhang, "Multiple-mode orthogonal frequency division multiplexing with index modulation," IEEE Transactions on Communications, vol. 65, no. 9, pp. 3892-3906, Sept. 2017.

[14] S. Dang, J. P. Coon, and G. Chen, "Adaptive OFDM with index modulation for two-hop relay-assisted networks," IEEE Transactions on Wireless Communications, vol. 17, no. 3, pp. 1923-1936, Mar. 2018.

[15] S. Dang, G. Chen, and J. P. Coon, "Lexicographic codebook design for OFDM with index modulation," IEEE Transactions on Wireless Communications, vol. 17, no. 12, pp. 8373-8387, Dec. 2018.

[16] J. Li, S. Dang, M. Wen, X. Jiang, Y. Peng, and H. Hai, "Layered orthogonal frequency division multiplexing with index modulation," IEEE Systems Journal, vol. 13, no. 4, pp. 3793-3802, Dec. 2019.

[17] S. Guo, S. Lv, H. Zhang, J. Ye, and P. Zhang, "Reflecting modulation," IEEE Journal on Selected Areas in Communications, vol. 38, no. 11, pp. 2548-2561, Nov. 2020.

[18] A. M. Jaradat, J. M. Hamamreh, and H. Arslan, "OFDM with subcarrier number modulation," IEEE Wireless Communications Letters, vol. 7, no. 6, pp. 914-917, Dec. 2018.

[19] S. Dang, G. Ma, B. Shihada, and M.-S. Alouini, "Enhanced orthogonal frequency-division multiplexing with subcarrier number modulation," IEEE Internet of Things Journal, vol. 6, no. 5, pp. 7907-7920, Oct. 2019.

[20] M. Elsaadany, A. Ali, and W. Hamouda, "Cellular LTE-A technologies for the future Internet-of-Things: Physical layer features and challenges," IEEE Communications Surveys Tutorials, vol. 19, no. 4, pp. 2544-2572, Fourthquarter 2017.

[21] S. Zhang, C. Lu, S. Jiang, L. Shan, and N. N. Xiong, "An unmanned intelligent transportation scheduling system for open-pit mine vehicles based on 5G and big data," IEEE Access, vol. 8, pp. 135 524-135 539, 2020.

[22] A. M. Jaradat, J. M. Hamamreh, and H. Arslan, "OFDM with hybrid number and index modulation," IEEE Access, vol. 8, pp. 55 042-55053, 2020.

[23] S. Dang, J. Zhou, B. Shihada, and M.-S. Alouini, "Relay assisted OFDM with subcarrier number modulation in multi-hop cooperative networks," 
IEEE Wireless Communications Letters, vol. 9, no. 11, pp. 1869-1873, Nov. 2020.

[24] M. Wen, J. Li, S. Dang, Q. Li, S. Mumtaz, and H. Arslan, "Jointmapping orthogonal frequency division multiplexing with subcarrier number modulation," IEEE Transactions on Communications, 2021 (early access article).

[25] J. Li, S. Dang, M. Wen, Z. Zhang, and Q. Li, "Smart detection using the cascaded artificial neural network for OFDM with subcarrier number modulation," IEEE Wireless Communications Letters, 2021 (early access article).

[26] J. M. Hamamreh, M. Kirik, M. O. Sagman, and N. Ishikawa, "Multiple input multiple output with antenna number modulation and adaptive antenna selection," RS Open Journal on Innovative Communication Technologies, vol. 4, pp. 1-14, June 2020.

[27] M. Kirik and J. M. Hamamreh, "Multiple MIMO with joint block antenna number modulation and adaptive antenna selection for future wireless systems," RS Open Journal on Innovative Communication Technologies, vol. 1, no. 2, pp. 1-15, Dec. 2020.

[28] S. Karatepe, M. Kirik, and J. M. Hamamreh, "Novel nonorthogonal multi-access method for multi-user MIMO with antenna number modulation," RS Open Journal on Innovative Communication Technologies, vol. 2, no. 3, pp. 1-11, Mar. 2021.

[29] M. Wen, X. Cheng, M. Ma, B. Jiao, and H. V. Poor, "On the achievable rate of OFDM with index modulation," IEEE Transactions on Signal Processing, vol. 64, no. 8, pp. 1919-1932, Apr. 2016.

[30] Z. Hu, J. Liu, and F. Chen, "On the mutual information and Huffman coding for OFDM-IM," in Proc. IEEE IMCEC, Xi' an, China, May 2018, pp. 298-302.

[31] M. I. Kadir, H. Zhang, S. Chen, and L. Hanzo, "Entropy coding aided adaptive subcarrier-index modulated OFDM," IEEE Access, vol. 6, pp. 7739-7752, 2018.

[32] M. E. Sahin, I. Guvenc, and H. Arslan, "Optimization of energy detector receivers for UWB systems," in Proc. IEEE VTC, vol. 2, Stockholm, Sweden, May 2005, pp. 1386-1390 Vol. 2.

[33] J. Proakis and M. Salehi, Digital Communications. McGraw-Hill, 2008

[34] E. Olfat and M. Bengtsson, "Joint channel and clipping level estimation for OFDM in IoT-based networks," IEEE Transactions on Signal Processing, vol. 65, no. 18, pp. 4902-4911, Sept. 2017.

[35] S. S. Das, E. De Carvalho, and R. Prasad, "Performance analysis of OFDM systems with adaptive sub carrier bandwidth,' IEEE Transactions on Wireless Communications, vol. 7, no. 4, pp. 1117-1122, Apr. 2008.

[36] J. Li, Q. Li, S. Dang, M. Wen, X. Jiang, and Y. Peng, "Low-complexity detection for index modulation multiple access," IEEE Wireless Communications Letters, vol. 9, no. 7, pp. 943-947, July 2020.

[37] Y. Yang and B. Jiao, "Information-guided channel-hopping for high data rate wireless communication," IEEE Communications Letters, vol. 12, no. 4, pp. 225-227, Apr. 2008.

[38] J. P. Coon, M. Badiu, Y. Liu, F. Yarkin, and S. Dang, "Binary-tree encoding for uniform binary sources in index modulation systems," IEEE Journal of Selected Topics in Signal Processing, vol. 13, no. 6, pp. 12701285 , Oct. 2019.

[39] T. Cover and J. Thomas, Elements of Information Theory. Wiley, 2012.

[40] S. Boyd and L. Vandenberghe, Convex Optimization. Cambridge University Press, 2004.

[41] S. Guo, H. Zhang, and M.-S. Alouini, "Asymptotic capacity for MIMO communications with insufficient radio frequency chains," IEEE Transactions on Communications, vol. 68, no. 7, pp. 4190-4201, July 2020.

[42] C. Liu, M. Ma, Y. Yang, and B. Jiao, "Optimal spatial-domain design for spatial modulation capacity maximization," IEEE Communications Letters, vol. 20, no. 6, pp. 1092-1095, June 2016.

[43] Z. Wang, S. Dang, S. Shaham, Z. Zhang, and Z. Lv, "Basic research methodology in wireless communications: The first course for researchbased graduate students," IEEE Access, vol. 7, pp. 86 678-86 696, 2019.

[44] Y. Liu and J. P. Coon, "Mitigating bit-synchronization errors in Huffmancoding-aided index modulation," IEEE Communications Letters, vol. 23, no. 3, pp. 426-429, Mar. 2019.

[45] D. S. Parker, Jr, "Conditions for optimality of the Huffman algorithm," SIAM Journal on Computing, vol. 9, no. 3, pp. 470-489, Aug. 1980.

[46] J. R. Hershey and P. A. Olsen, "Approximating the Kullback Leibler divergence between gaussian mixture models," in Proc. IEEE ICASSP, vol. 4, Honolulu, HI, USA, Apr. 2007, pp. 317-320.

[47] J. M. Hamamreh, A. Hajar, and M. Abewa, "Orthogonal frequency division multiplexing with subcarrier power modulation for doubling the spectral efficiency of 6G and beyond networks," Transactions on Emerging Telecommunications Technologies, vol. 31, no. 4, pp. 1-18, Apr. 2020. 